\title{
Equidad de género del sistema tributario en la Argentina: estimación de la carga fiscal desglosada por tipo de hogar ${ }^{1}$
}

\author{
Darío Rossignolo
}

\section{Resumen}

El objetivo de este artículo es incorporar la dimensión de género en el análisis de la incidencia tributaria. Para ello se calculan los efectos de los impuestos directos e indirectos en la distribución del ingreso según el género en la Argentina, con miras a establecer el grado de progresividad de los impuestos y su efecto en la equidad de género conforme la clasificación de los hogares. Los resultados muestran que mientras el sistema tributario es moderadamente progresivo y la mayor carga recae sobre los hogares con hombres como perceptores de ingreso, surgen diferencias al considerar impuestos indirectos y directos por separado. Los primeros son fuertemente regresivos y los hogares con mujeres como perceptoras de renta soportan la mayor carga por estar concentrados en los tramos de ingreso más bajos. Los hogares con niños soportan la mayor carga de impuestos directos, particularmente aquellos en los que existen hombres perceptores y perceptores duales.

\section{Palabras clave}

Política fiscal, tributación, género, hogares, ingresos, igualdad de género, Argentina

\section{Clasificación JEL}

$\mathrm{H} 2, \mathrm{I3}, \mathrm{D} 3$

\section{Autor}

Darío Rossignolo es Profesor del Departamento de Economía de la Facultad de Ciencias Económicas en la Universidad de Buenos Aires. Correo electrónico: darossignolo@gmail.com.

\footnotetext{
1 Varios resultados de este trabajo se basan en Rossignolo (2016).
} 


\section{Introducción}

Uno de los principales elementos necesarios para el diseño de la política fiscal es el conocimiento de la manera en que la cantidad de impuestos pagados se distribuye entre los diferentes niveles de ingresos. Un sistema tributario satisface la "equidad vertical" cuando los sectores con mayor capacidad contributiva pagan una mayor proporción de impuestos con respecto a los demás, mientras que la "equidad horizontal" define el hecho de que los sectores con el mismo nivel de bienestar o capacidad de pago deberían pagar la misma proporción de impuestos (Lambert, 1993).

Los sistemas tributarios tienen importantes repercusiones en la equidad social y de género. El punto de partida del argumento es que la recaudación de impuestos eficaz es una condición necesaria, pero no suficiente, para mejorar la desigualdad y la pobreza por razones de género. Una recaudación impositiva agregada baja tiene consecuencias en la equidad de género porque impide el establecimiento de programas que contrarresten la distribución del ingreso del mercado, en el que generalmente las mujeres resultan desfavorecidas. Esto se debe a que realizan la mayor parte del trabajo de cuidado no remunerado (pues su trabajo remunerado tiene lugar en el sector informal en mayor medida que el trabajo remunerado de los hombres) y a que, en caso de trabajar en el sector formal, tienden a estar empleadas en empresas más pequeñas y a ganar menos que los hombres (Grown y Valodia, 2010).

El análisis de género estudia los efectos de los impuestos y las políticas fiscales en el bienestar dentro de los hogares. El objetivo de este trabajo es analizar los efectos del sistema tributario en la equidad de género en la Argentina. El análisis se basa en la Encuesta Nacional de Gastos de los Hogares (ENGHo), realizada por el Instituto Nacional de Estadística y Censos (INDEC) de la Argentina desde marzo de 2012 a febrero de 2013, de manera que se emplean los códigos tributarios pertinentes para 2012.

El estudio se estructura de la siguiente manera: en la segunda sección se presentan algunas consideraciones teóricas sobre los análisis de incidencia y la evaluación de género de los sistemas tributarios. Asimismo, se describe brevemente el contexto argentino en términos de dimensiones de género y se examinan los resultados de algunos estudios anteriores sobre este tema. En la tercera sección se describe el contexto tributario general y se muestra la composición de los ingresos tributarios y su evolución. En la cuarta sección se presentan el marco conceptual del análisis de los efectos de los impuestos dentro de los hogares, el marco jurídico del sistema tributario en la Argentina y la metodología para el cálculo de la incidencia económica y de género. En la quinta sección se detallan los resultados del análisis de incidencia en la desigualdad de ingresos y de género y en la sexta sección se realiza un resumen y se formulan las conclusiones.

\section{Consideraciones teóricas generales y antecedentes empíricos}

De conformidad con la literatura sobre equilibrio parcial, el análisis de incidencia tributaria realizado en este trabajo se conoce como enfoque contable y procura analizar quién paga los impuestos al Estado. El concepto de "incidencia" utilizado en este documento se refiere a la cantidad de impuestos pagados por cada hogar. Si bien en algunos casos esa información puede obtenerse directamente a partir de encuestas por muestreo, puede ser necesario realizar algunas inferencias. Cuando los impuestos no figuran directamente en las encuestas se deberá establecerlos de manera indirecta. De acuerdo con Bourguignon y Da Silva (2003), los métodos indirectos suponen la aplicación de escalas impositivas oficiales o la imputación de impuestos indirectos pagados mediante gastos observados. 
Sin embargo, los enfoques contables dejan de lado posibles respuestas conductuales de los agentes que pueden modificar las cantidades que realmente pagan o reciben. Por ejemplo, un enfoque contable no detectaría la evasión fiscal derivada de un incremento en los tipos impositivos sobre la renta. Estos enfoques se limitan a los efectos de primera ronda y no tienen en cuenta los efectos de segunda ronda atribuibles a respuestas conductuales, que los enfoques conductistas tratan de tener en consideración.

De manera ideal, el cálculo de la incidencia tributaria, en el caso de los impuestos sobre bienes, debería suponer la estimación de la variación compensatoria, que permitiría establecer cuánto disminuye el ingreso real como consecuencia del impuesto. De acuerdo con Sahn y Younger (2003), los métodos se aproximan a ese cálculo mediante la variación compensatoria. La variación compensatoria de un cambio marginal en el precio de un bien es simplemente el cambio en el presupuesto de consumo necesario para mantener la canasta de consumo constante. En otras palabras, en una primera aproximación es posible ignorar la respuesta de la demanda al impuesto (Sahn y Younger, 2003).

Para elaborar metodologías coherentes con los antecedentes teóricos, en este estudio se asume que la carga generada por los impuestos sobre los bienes y los servicios se transfiere completamente a los consumidores y se consideran los tipos reglamentarios sobre cada una de las partidas de gastos examinadas. Por el contrario, la carga fiscal por concepto de impuestos directos se traslada hacia atrás hacia la fuente de ingresos, mediante la reducción de los ingresos disponibles para los perceptores de ingresos.

La variable clave para analizar los impuestos pagados por cada quintil y categoría de hogares es la carga tributaria, es decir la proporción de impuestos con respecto a los ingresos, per cápita y antes de impuestos. En consecuencia, un impuesto será progresivo si la proporción aumenta al considerar niveles de bienestar mayores y, por el contrario, será regresivo si la carga tributaria disminuye con el ingreso per cápita (mediante significación estadística).

\section{Análisis del sesgo de género en los sistemas tributarios}

En su análisis de la existencia de sesgos de género en los sistemas tributarios y, en particular, del impuesto sobre la renta de las personas físicas, Stotsky (1996) señala que este tipo de discriminación puede tener lugar de manera explícita, implícita o ambas. Mientras que el sesgo de género explícito se encuentra en muchos sistemas tributarios porque depende del lenguaje utilizado en las normas o el código tributario, el sesgo de género implícito es más difícil de determinar, pues depende de las diferentes repercusiones que estas normas y disposiciones legales tienen para hombres y mujeres, que dependen de juicios de valor con respecto al comportamiento social y económico deseable. Aunque los sistemas de declaración individual pueden ser en general más equitativos que los de declaración conjunta desde el punto de vista del género, a menudo todavía contienen sesgos de género explícitos e implícitos.

De acuerdo con Grown (2010), la asignación de deducciones, exenciones y otros privilegios fiscales sobre la base del sexo constituye un ejemplo de sesgo de género explícito. Por ejemplo, las deducciones pueden ser diferentes si el perceptor de ingresos está en el sector formal o es un trabajador independiente. Debido a que los hombres tienen más probabilidades de ser empleados y menos probabilidades de estar a cargo de un hogar monoparental que las mujeres, es más probable que estos se encuentren en hogares con una carga menor de impuesto sobre la renta de las personas físicas. Estas disposiciones legales constituyen sesgos implícitos contra las mujeres, que predominan entre los trabajadores por cuenta propia al frente de hogares monoparentales. El código tributario también prevé exenciones para los pagos de intereses o dividendos. Si se tiene en cuenta que la 
probabilidad de poseer acciones y títulos es mayor para los hombres que para las mujeres, esto constituye otra forma de sesgo de género implícito.

Al examinar los impuestos indirectos es necesario tener en cuenta un factor no considerado en el debate acerca del impuesto sobre la renta de las personas físicas: las diferencias de género en las modalidades de consumo. Podría ser, por ejemplo, que la incidencia de los impuestos indirectos fuera de hecho inferior en los hogares encabezados por mujeres que en los demás, porque las mujeres tienden a comprar y consumir menos productos de las categorías sujetas a los impuestos indirectos más altos (por ejemplo, alcohol y tabaco).

Stotsky (1996) argumentaría que un sistema de impuestos indirectos en que los impuestos sobre el alcohol son más altos que los de muchos otros productos está sesgado en contra de los hombres. A juicio de esta autora, en un sistema imparcial los bienes y servicios consumidos de manera desproporcionada por los hombres no deberían gravarse con un tipo impositivo diferente del de los bienes consumidos de manera desproporcionada por las mujeres. Del mismo modo, los tipos más altos del impuesto sobre el valor agregado (IVA) a la asistencia sanitaria están implícitamente sesgados contra las mujeres, que son las principales consumidoras en ese caso.

Este argumento deriva de la opinión de Stotsky de que el sesgo proviene del tratamiento diferenciado de mujeres y hombres, que en realidad deberían ser tratados de la misma manera (es decir, se viola el principio de equidad horizontal). Sin embargo, esto supone que el consumo de alcohol y tabaco y el consumo de asistencia sanitaria son formas de comportamiento igualmente valiosas desde el punto de vista social e igualmente una cuestión de elección personal, productora de utilidades².

Sin embargo, en general se considera justificable gravar los bienes de valor social y las necesidades básicas a un tipo impositivo inferior con respecto a los bienes que carecen de valor social y los artículos de lujo. En consecuencia, si los bienes consumidos de manera desproporcionada por los hombres son bienes que carecen de valor social, de lujo o ambas cosas a la vez y los bienes consumidos de manera desproporcionada por las mujeres son bienes de valor social, necesidades básicas o ambas cosas a la vez, el establecimiento de tipos más altos para los primeros (y de tipos más bajos para los últimos) no violaría el principio de equidad entre mujeres y hombres. El principio pertinente en este caso es el de equidad vertical, que permite que las personas en diferentes situaciones sean tratadas de manera adecuadamente distinta.

A la luz del probable efecto de los dos impuestos en la conducta de las mujeres y los hombres y de la posibilidad de que los hombres transfieran la carga de los impuestos sobre el alcohol a otros miembros del hogar, este aspecto requiere un análisis más profundo. Por lo general, los consumidores de alcohol no reducen sus compras de este producto cuando el precio aumenta, de manera que existe el riesgo de que los hombres respondan a un incremento en el precio del alcohol recortando los gastos en bienes que benefician a otros miembros del hogar. Del mismo modo, en caso de un aumento del IVA sobre los artículos de primera necesidad (por ejemplo jabón, sal, queroseno), de cuya compra son responsables las mujeres, la evidencia sugiere que estas tienden a recortar su propio consumo y a dedicar una mayor parte de su tiempo a producir sustitutos caseros.

Existe una considerable evidencia de que el poder de negociación de las mujeres dentro de los hogares tiende a ser menor que el de los hombres (Doss, 2011). Así, es plausible es que los hombres tengan una mayor capacidad que las mujeres para trasladar la carga de los impuestos indirectos al consumo (como los impuestos sobre consumos específicos y el IVA) a otros miembros del hogar. De ser así, los impuestos indirectos al consumo tendrán efectos muy desiguales en los hombres y las mujeres, con mayores repercusiones entre estas últimas.

\footnotetext{
2 Sería recomendable una investigación actualizada de estos temas para confirmar la persistencia de estas modalidades de consumo en diferentes sociedades.
} 


\section{Análisis del sesgo de género en el código tributario del impuesto sobre la renta de las personas físicas argentino ${ }^{3}$}

En la Argentina se ha adoptado el principio de declaración individual: independientemente de su estado civil, cada contribuyente debe presentar una declaración de impuestos personal (si corresponde) y pagar impuestos sobre la renta imponible 4 . Los ingresos derivados de actividades personales, bienes muebles y bienes inmuebles adquiridos con los frutos de la profesión o el empleo del cónyuge, entre otros, se deben asignar a cada uno de los cónyuges.

Sin embargo, en un régimen tributario individual es posible encontrar sesgos de género en tres casos distintos. En primer lugar, en la asignación de los ingresos no laborales. El ingreso por concepto de empleo se asigna al empleado, pero la asignación de los ingresos de otras fuentes, como el ingreso derivado de bienes inmuebles, no está tan clara. Este tipo de ingreso suele asignarse de distintas maneras en los sistemas tributarios de diferentes países, a saber: se asigna al cónyuge con mayores ingresos, se distribuye equitativamente entre los cónyuges, se permite que los cónyuges decidan sobre su asignación o se asigna al cónyuge propietario del bien inmueble que lo genera.

En segundo lugar, las exenciones de impuestos pueden aplicarse de manera distinta según el régimen tributario empleado. Las disposiciones tributarias generalmente admiten exenciones fiscales por distintos motivos, incluida la declaración de niños pequeños y cónyuges a cargo. En un régimen de declaración de impuestos individual, la necesaria distribución de esas exenciones entre los cónyuges puede no ser justa, por ejemplo, en los casos en que se prevén exenciones para los maridos con esposas a cargo, pero no para las esposas con maridos a cargo. En tercer lugar, en algunos países se aplican distintos tipos impositivos a los hombres y las mujeres y los tipos impositivos para las mujeres casadas son más altos que aquellos para los hombres casados.

De esas tres posibles formas de discriminación, la tercera no se aplica al caso argentino porque los hombres y las mujeres están sujetos a los mismos tipos impositivos. Sin embargo, las dos primeras deberían evaluarse en función de la aplicación del impuesto sobre la renta de las personas físicas. Esto se debe a que, en el caso de las parejas casadas, varias exenciones asignan al marido determinadas fuentes de ingresos comunes (por ejemplo, los beneficios derivados de bienes gananciales se asignan completamente al marido en casi todos los casos). En la Argentina, los ingresos derivados de los bienes gananciales deben registrarse en la declaración de impuestos del marido.

En general se acepta que el régimen aplicable no es exactamente el régimen de tributación individual que aparenta ser, sino más bien un régimen híbrido, pues incluye casos en que los cónyuges están sometidos a una especie de tributación conjunta. Sin embargo, cabe señalar que este sesgo no supone una penalización económica para las mujeres.

\section{Contexto de equidad de género en la Argentina}

En la Argentina, la población crece a una tasa media anual del 1,1\% y la organización familiar cambia muy lentamente. Se proyecta que a partir de 2040 la población del país crecerá a una tasa inferior al $1 \%$ anual acumulado. Esto se debe a la reducción del tamaño medio de la familia porque las parejas tienen menos hijos, al aumento de los hogares monoparentales, a la disminución relativa de las familias numerosas y compuestas y a la correlativa predominancia de la familia nuclear. Los hogares nucleares

\footnotetext{
3 Esta sección se basa en Rodríguez Enríquez, Gherardi y Rossignolo (2010).

4 Como se verá más adelante, el impuesto sobre la renta de las personas físicas puede pagarse mediante declaraciones de impuestos o retenciones sobre salarios y sueldos o mediante el régimen simplificado de monotributo.
} 
continúan predominando en la Argentina (57,5\%) y la mayoría $(85,8 \%)$ de ellos está encabezada por un hombre. En contraste, las mujeres encabezan el $58,7 \%$ de los hogares con un solo miembro y el $75,5 \%$ de los hogares monoparentales ${ }^{5}$.

El desempleo llegó al 7,6\% en 2013 (un 8,5\% en el caso de los hombres y un 11,6\% en el de las mujeres). Al tener en cuenta la dimensión de género, el desempleo es mayor para las mujeres adultas (15-64 años) que para los hombres (el 6,9\% y el 4,5\%, respectivamente, en 2013), mientras que entre los jóvenes (15-24 años), el desempleo aumenta al 24\% de la fuerza de trabajo entre las mujeres y al $18 \%$ entre los hombres.

La proporción de trabajadores informales se mantuvo en niveles superiores al 39\% de la población económicamente activa (PEA) en 2013. El 42,4\% de las mujeres tiene un empleo informal, mientras que en el caso de los hombres esa cifra es del $36,8 \%{ }^{6}$. En consecuencia, las mujeres entran y salen de la fuerza de trabajo con mayor frecuencia que los hombres, de manera que su participación es más discontinua y tienen más probabilidades de realizar trabajos a tiempo parcial y de temporada que los hombres, que se concentran en los puestos a tiempo completo.

Como se muestra en el cuadro 1, en la Argentina la fuerza de trabajo es mayor y más estable en el caso de los hombres que en el de las mujeres. Esta situación no es homogénea en los distintos niveles de ingresos, pues la participación de las mujeres de bajos ingresos en la fuerza de trabajo es mucho menor que la de sus pares con altos ingresos. Esto significa que la brecha de género es mayor para cada quintil de ingresos más bajos (Rodríguez Enríquez, Gherardi y Rossignolo, 2010). La presencia de niños pequeños en el hogar continúa siendo una de las principales barreras a la entrada de las mujeres en el mercado de trabajo, situación que se acentúa a medida que disminuye el nivel de ingresos del hogar.

En segundo lugar, los ingresos individuales de las mujeres son menores que los de los hombres. Si bien las estadísticas oficiales muestran que la brecha está disminuyendo, el ingreso laboral de las mujeres es todavía inferior a alrededor del $60 \%$ del ingreso de los hombres. Al tener en cuenta los ingresos de todas las fuentes, el porcentaje aumenta al 70\%. Esto demuestra que los ingresos de los hombres están más concentrados en el empleo formal que los de las mujeres (véase el gráfico 1). El nivel relativamente más bajo de ingresos percibidos por las mujeres con respecto a los hombres, es decir, la brecha constante en términos de ingresos, puede explicarse por el mayor nivel de desempleo, la inestabilidad del empleo y la mayor concentración de las mujeres en los trabajos menos formales.

5 Base de datos socioeconómicos para América Latina y el Caribe (Centro de Estudios Distributivos, Laborales y Sociales (CEDLAS) y Banco Mundial).

6 Base de datos socioeconómicos para América Latina y el Caribe (Centro de Estudios Distributivos, Laborales y Sociales (CEDLAS) y Banco Mundial). De acuerdo con la definición de informalidad empleada en este trabajo, son informales todos los trabajadores asalariados de pequeñas empresas, los trabajadores no profesionales por cuenta propia y los trabajadores con cero ingresos. 
8

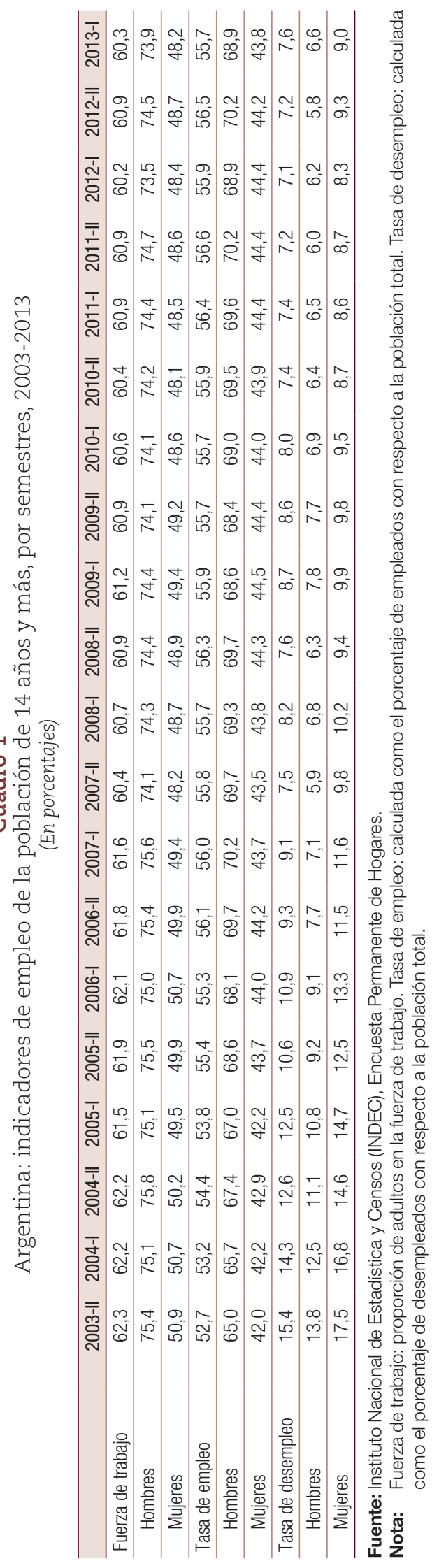




\section{Gráfico 1}

Argentina: ingresos de las mujeres como porcentaje de los ingresos de los hombres, 2004-2013

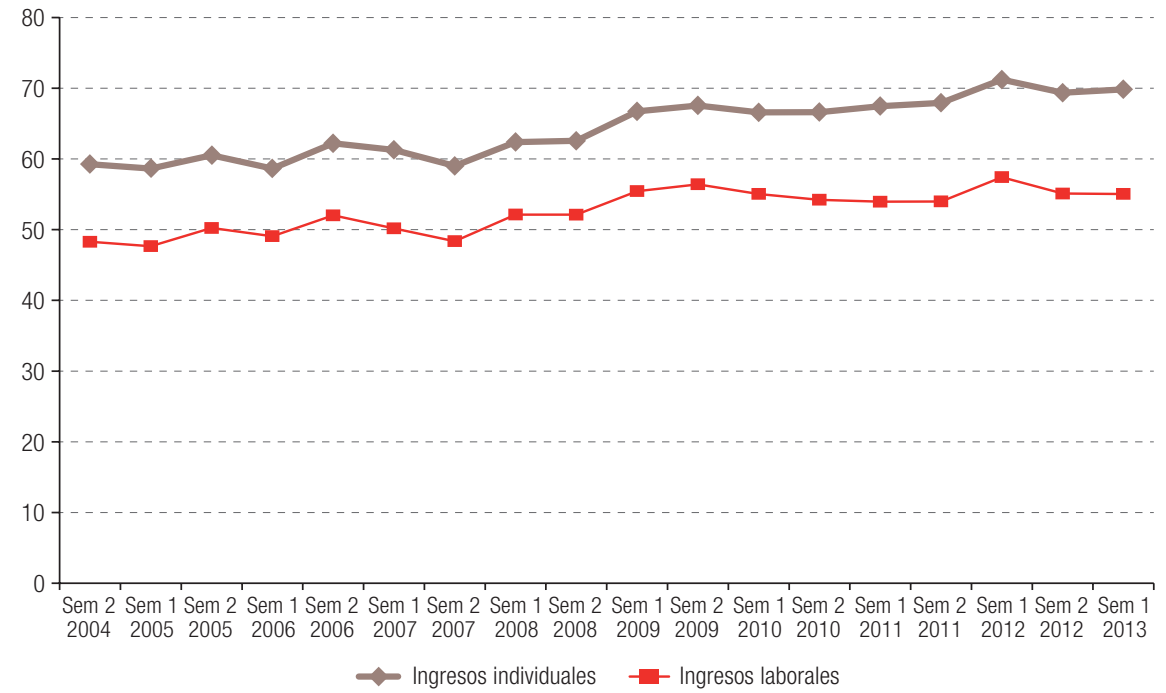

Fuente: Elaboración propia, sobre la base de datos del Instituto Nacional de Estadística y Censos (INDEC), Encuesta Permanente de Hogares.

En tercer lugar, las mujeres trabajan predominantemente en empleos informales de manera que resultan excluidas de la red del impuesto sobre la renta (Grown, 2010). El perfil de empleo de las mujeres (trabajo discontinuo, ingresos relativos menores y predominancia en el empleo informal) determinan su falta de acceso a los beneficios otorgados a los empleados formales mediante el sistema tributario.

En la Argentina, las mujeres no solo disponen de menos puestos de trabajo que los hombres, sino que también continúan concentrándose en mayor medida en empleos de baja calidad ${ }^{7}$. Las mujeres están sobrerrepresentadas: i) en el trabajo a tiempo parcial, ii) entre los trabajadores asalariados informales, iii) en los empleos que requieren menos calificación; iv) en el trabajo temporal o por un período específico, y v) en los servicios domésticos (Rodríguez Enríquez, Gherardi y Rossignolo, 2010).

Además de disponer de menos opciones de empleo que los hombres, las mujeres continuaron estando sobrerrepresentadas en los trabajos de peor calidad y menos calificados. Mientras el $36,8 \%$ de los trabajadores asalariados desempeñaban trabajos informales en 2013, esta proporción aumentaba al $42,4 \%$ en el caso de las mujeres. Con respecto a los trabajos menos calificados, persiste la segregación en ocupaciones específicas. La proporción de mujeres en los servicios sociales asciende al $44,1 \%$, mientras que los hombres todavía predominan en los sectores de la construcción y manufacturero. Además, los servicios domésticos continuaron siendo la principal fuente de empleo femenino. De hecho, este sector comprende al $17,2 \%$ de las mujeres activas y al $22,7 \%$ de las trabajadoras asalariadas. Esta sigue siendo una opción laboral sumamente precaria.

Las razones descritas explican la brecha de ingresos en el trabajo remunerado mencionada anteriormente. Esta brecha deriva de la combinación de los distintos tipos de discriminación señalados (menor participación, mayor desempleo y subempleo, segregación horizontal y vertical), a los que se suma el hecho de que las mujeres trabajan menos horas que los hombres debido a varios factores limitantes, como la presencia de niños pequeños en el hogar.

\footnotetext{
7 En 2013, el trabajo no remunerado representó el 33\% del tiempo de trabajo de los hombres y el 73\% del tiempo de trabajo de las mujeres (CEPALSTAT).
} 
El hecho de que las mujeres tengan más dificultades que los hombres para lograr la plena participación en el mercado laboral se traduce en una mayor vulnerabilidad desde el punto de vista de sus ingresos. Esto cobra aún más importancia al tener en cuenta que las mujeres están sobrerrepresentadas en los tipos de hogares con mayores niveles de precariedad, por ejemplo, los hogares monoparentales. En estos casos, los ingresos de los hogares encabezados por hombres son casi un 30\% más altos que los de los hogares encabezados por mujeres. Todo esto se traduce, a su vez, en una capacidad contributiva diferenciada entre hombres y mujeres que, en consecuencia, incide en la estructura tributaria actual de diferentes maneras.

Asimismo, existen diferencias en las modalidades de gastos de consumo entre los tipos de hogares. En comparación con los hombres, las mujeres tienden a gastar una mayor proporción de los ingresos bajo su control en bienes como alimentos, educación y asistencia sanitaria, que aumentan el bienestar y las capacidades de los niños. Estas modalidades afectan la incidencia de los impuestos.

\section{Resultados de estudios anteriores del caso argentino}

Si bien existen algunos antecedentes sobre la incidencia tributaria en la Argentina, son muy escasos los estudios realizados en que se tuvo en cuenta la perspectiva de género. Gasparini (1998) analiza el efecto distributivo del sistema tributario en 1996 sobre la base de los ingresos per cápita y los gastos de consumo per cápita como indicadores de bienestar. En el primer caso, los impuestos son muy regresivos, mientras que al considerar el consumo per cápita la incidencia resulta moderadamente progresiva. Gómez Sabaini, Santiere y Rossignolo (2002) analizan los efectos de los impuestos en la distribución del ingreso en 1997, teniendo en cuenta los ingresos per cápita ajustados por subregistro como medida de bienestar. La incidencia en este caso es regresiva y el IVA y los impuestos indirectos encabezan dicho efecto.

Gómez Sabaini, Harriague y Rossignolo (2013) examinan la incidencia det los impuestos en 2008, también sobre la base de los ingresos per cápita. Aquí, el efecto de los impuestos varía de proporcional a ligeramente progresivo, sobre todo debido a los derechos de exportación y al aumento de la importancia del impuesto sobre la renta y los impuestos sobre la nómina, medido por el coeficiente de Gini. Sin embargo, las diferencias en los extremos (es decir, entre el décimo decil y el primer decil) aumentan y esto permite concluir que, en ese caso, persiste la característica regresiva del sistema.

El trabajo de Rodríguez Enríquez, Gherardi y Rossignolo (2010) constituye el único antecedente de un análisis de incidencia con perspectiva de género. El análisis realizado cubre solo los impuestos indirectos a nivel nacional, utiliza los gastos de consumo per cápita como indicador de bienestar y se basa en datos de 2005. Los resultados muestran que la mayor carga fiscal es relativamente más alta para los hogares con dos fuentes de ingresos, los hogares con un sostén de la familia masculino y los hogares integrados mayoritariamente por hombres. El efecto en la distribución de los impuestos indirectos se explica por la existencia simultánea de una cierta regresividad del IVA, una marcada regresividad de los impuestos sobre consumos específicos y una acentuada progresividad del impuesto sobre los combustibles. De hecho, el primer quintil de gasto tiene una carga de IVA mayor que la de los otros quintiles, con respecto a todos los tipos de hogares.

\section{Estructura tributaria general en la Argentina}

El sector público argentino se caracteriza por una larga historia de desequilibrios estructurales (Gómez Sabaini y Rossignolo, 2009). En los últimos diez años, el superávit del sector público se redujo de una media del 3,2\% del producto interno bruto (PIB) entre 2004 y 2008 a una media del 0,5\% entre 2009 y 2013, mientras que el superávit primario representó una media del 1,6\% y un déficit medio del 1,2\%, 
respectivamente, en los mismos períodos. Si bien la carga fiscal aumentó en forma constante (como se verá en la próxima sección), el aumento del gasto público fue aún mayor y determinó que a partir de 2012 prevaleciera el déficit en los dos niveles de agregación.

En la Argentina se ha registrado un considerable incremento de la carga fiscal en la última década, que llegó al 31,2\% del PIB en $2013^{8}$. Esto se relaciona con la adición de impuestos que se utilizaron esporádicamente en períodos anteriores, como los derechos de exportación (retenciones) y transacciones en cuenta corriente, y con la aplicación de otras disposiciones que afectaron el impuesto de sociedades y el impuesto sobre la renta de las personas físicas (sin ajustes de inflación de umbrales y estados financieros). Esto se puede observar en el gráfico 2, en el que, según la clasificación de los impuestos del Fondo Monetario Internacional (FMI), el impuesto sobre las transacciones bancarias se incluye en los impuestos sobre la propiedad. Si bien los derechos de exportación se han vuelto importantes, su importancia relativa ha disminuido debido a la reducción de los precios internacionales de los productos básicos.

\section{Gráfico 2}

Argentina: carga fiscal bruta nacional y provincial, 2004-2013

(Como porcentaje del PIB)

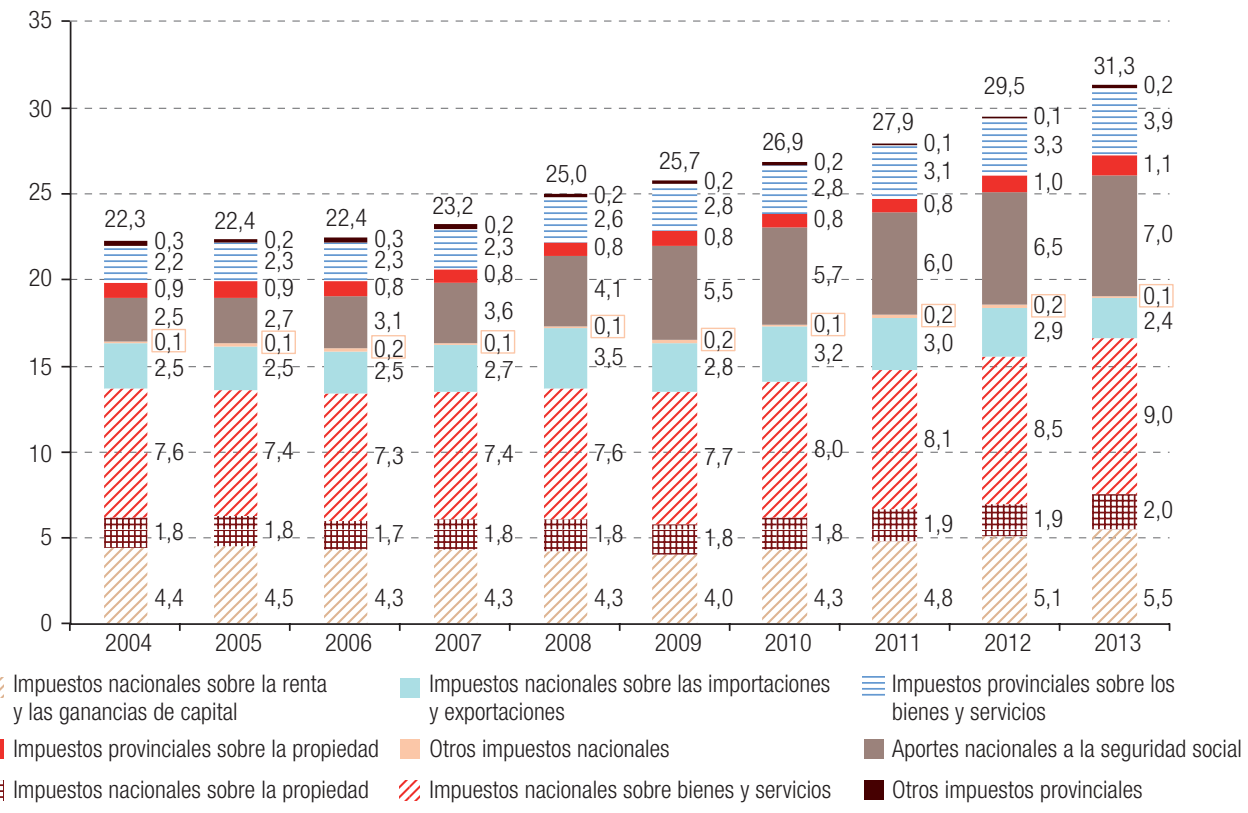

Fuente: Ministerio de Hacienda y Finanzas Públicas.

La mayor parte de esta carga fiscal corresponde a impuestos indirectos. Sin embargo, la participación relativa de las diferentes clases de impuestos ha cambiado ligeramente. Como se puede observar en el gráfico 3, casi el $42 \%$ de la recaudación tributaria provino de los impuestos indirectos en 2013, combinando la recaudación de impuestos nacionales y provinciales sobre bienes y servicios. Entre ellos, la proporción de los impuestos generales sobre el consumo (principalmente el IVA) continúa siendo superior (alrededor del 60\%). Al considerar exclusivamente los impuestos sobre consumos específicos, la mayor proporción (alrededor del 60\%) corresponde al impuesto sobre los combustibles, seguidos por el tabaco (24\%), pese a que su participación ha disminuido.

8 Carga fiscal bruta, no incluye reembolsos. Los ingresos derivados de las contribuciones al seguro de salud se sumaron a los ingresos por impuestos, siempre que sean obligatorios para los trabajadores del sector formal. Sin embargo, estas cifras son considerablemente inferiores a las disponibles antes del nuevo cálculo oficial del PIB (con 1993 como año de base). Estas nuevas cifras redujeron el PIB nominal oficial (con 2004 como año de base) y disminuyeron las cifras relativas a la carga fiscal en aproximadamente un $5 \%$ del PIB. 


\section{Gráfico 3}

Argentina: composición de los impuestos nacionales y provinciales, 2004-2013

(En porcentajes)

\section{A. Impuestos indirectos}

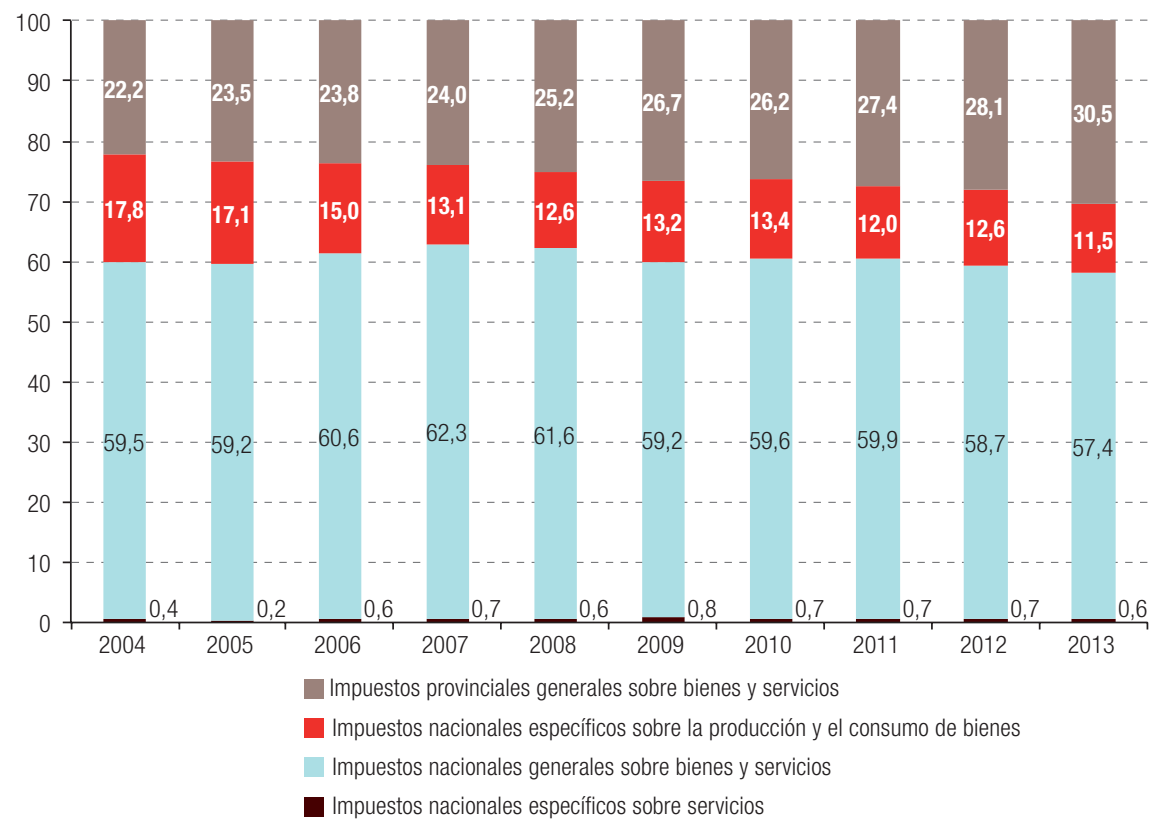

B. Impuestos directos

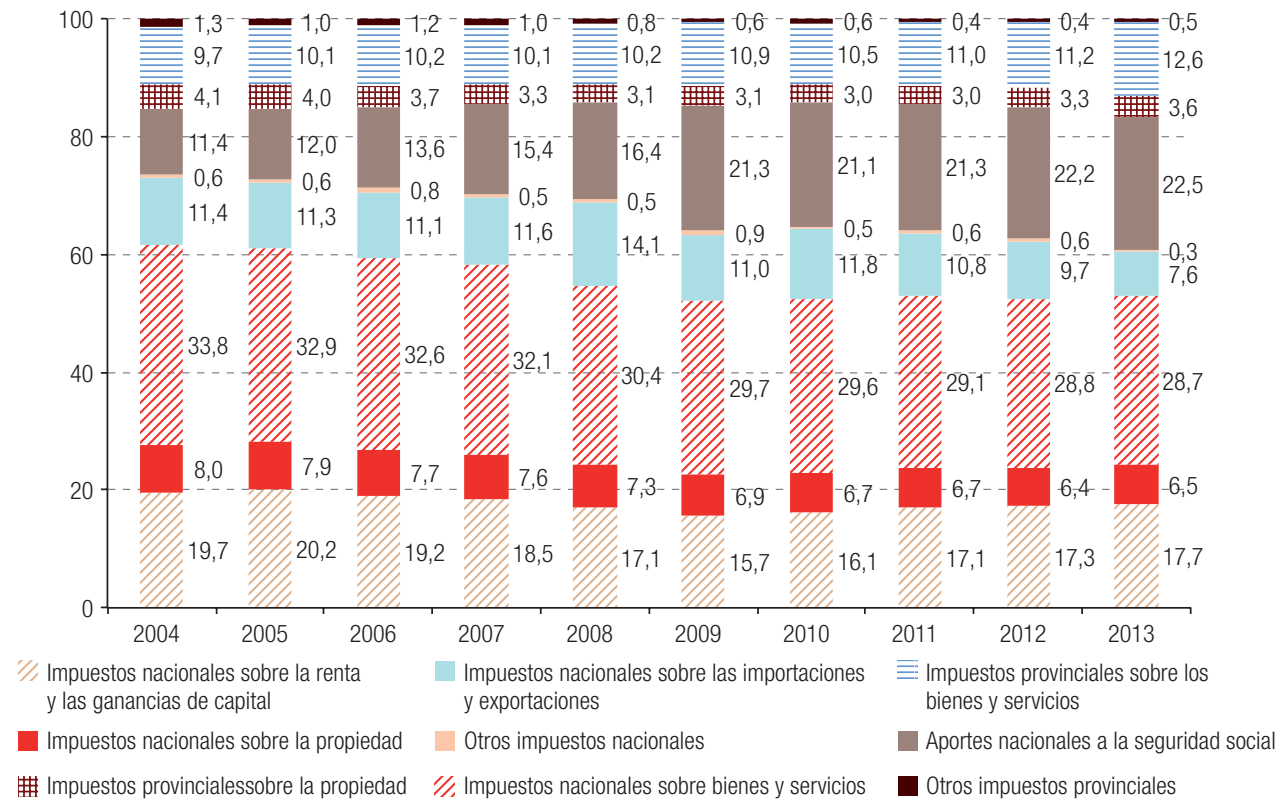

Fuente: Ministerio de Hacienda y Finanzas Públicas.

En 2008, los ingresos por concepto de aportes a la seguridad social cobraron importancia, al constituir la mayor fuente de ingresos de impuestos directos. Esto se debió a la eliminación del sistema de capitalización implementado en la década de 1990, de manera que todos esos recursos fueron captados por el Gobierno para el establecimiento del sistema de reparto. 
En resumen, el incremento de la carga fiscal puede explicarse en gran medida por una gran cantidad de impuestos que podrían considerarse extraordinarios o de emergencia. Cabe señalar que en esta década también se hizo hincapié en medidas de administración tributaria. Resulta evidente que las reformas tributarias argentinas estuvieron más orientadas a evitar el desequilibrio fiscal que a incrementar la equidad de género.

\section{Análisis de incidencia tributaria: consideraciones metodológicas}

En este trabajo no solo se actualiza la investigación anterior sobre el tema utilizando información de la ENGHo relativa a 2012-2013, sino que también se incluyen los impuestos indirectos a nivel provincial. Asimismo, se realizaron cálculos teniendo en cuenta los ingresos per cápita como indicador de bienestar, así como la estimación de la incidencia de los impuestos directos.

La principal fuente de información para este informe fue la ENGHo, que es una encuesta a gran escala mediante la cual se obtienen respuestas detalladas de alrededor de 20.960 hogares de todo el país (alrededor de 36,1 millones de habitantes) ${ }^{9}$. Las unidades analizadas por la encuesta corresponden a hogares individuales. Las principales variables de estudio de la encuesta son los gastos y los ingresos de los hogares. La encuesta también incluye información sobre variables demográficas, laborales y educativas, además de características de la vivienda, transferencias en especie recibidas y bienes del hogar ${ }^{10}$.

\section{Distribución del indicador de bienestar}

En los estudios de incidencia tradicionales las unidades de análisis se clasifican sobre la base de los ingresos actuales. Sin embargo, si una persona modifica su consumo en distintos períodos, su bienestar estará mejor relacionado con los ingresos permanentes. Esto se resuelve al clasificar a las personas de acuerdo con sus ingresos permanentes o su mejor sustituto, el consumo.

Desde una perspectiva de género, sería más interesante determinar los efectos de los impuestos en los hogares más pobres, debido a que las probabilidades de formar parte de hogares de bajos ingresos son mayores para las mujeres que para los hombres. En consecuencia, los ingresos se considerarán como indicador de bienestar, a pesar de las enormes dificultades que se enfrentan en las encuestas de hogares para recoger valores precisos para esa variable, que pueden generar dudas sobre la fiabilidad de los datos presentados. Por consiguiente, en los ingresos se incluyen los ingresos de los trabajadores asalariados, empleadores, trabajadores independientes, perceptores de ganancias de capital, beneficiarios de la seguridad social, pensionistas y beneficiarios de transferencias públicas.

El objetivo es captar la distribución del ingreso antes de impuestos. Los ingresos indicados en las encuestas de hogares incluyen transferencias públicas, que constituyen una medida después de impuestos (ajustada por las diferencias entre los precios regionales). Para calcular los ingresos antes de impuestos las transferencias públicas (monetarias y no monetarias) deberían excluirse de los ingresos declarados. Las transferencias públicas mencionadas incluyen las pensiones. En algunos

9 La ENGHo es una muestra representativa del 86,8\% de la población (se excluyen solamente las ciudades con menos de 5.000 habitantes). Para llegar a la población total del país, es necesario tener en cuenta el porcentaje de la población urbana que no está incluida en la muestra, así como la información sobre las áreas rurales, que se excluyeron explícitamente de la muestra debido a los altos costos operativos que suponía su utilización en una encuesta. No hay declaraciones oficiales de las autoridades sobre la fiabilidad de esta encuesta.

10 Véase la definición de ingresos y gastos incluida en la encuesta en INDEC (2013). 
casos, cuando la pensión es el único ingreso que percibe una persona, al restar este ítem el hogar resulta sin ingresos. Esto significaría que el hogar paga impuestos y compra artículos a pesar de no tener ingresos, exagerando el efecto de las pensiones. Para evitar estos inconvenientes, las pensiones públicas se mantuvieron en la definición de ingresos disponibles, considerándolas como parte de los ingresos antes de impuestos.

Esto se aplica a las transferencias monetarias y no monetarias. Excluir estas transferencias de los ingresos significaría que las personas no utilizan los ingresos de esta fuente para el consumo y en consecuencia pagan impuestos. Sin embargo, debido a que en la encuesta de hogares no se distinguen las transferencias que recibe el hogar, estas se mantuvieron en la definición de ingresos y en el indicador de bienestar. Esta definición se utilizó para calcular los quintiles y para todos los análisis de incidencia.

\section{Análisis de género mediante una tipología de hogares}

Para realizar el análisis de género, los quintiles definidos anteriormente se dividieron en diferentes categorías según la composición del hogar dentro de cada quintil. La clasificación presentada en este trabajo se basa en las características laborales de sus miembros y tiene el objetivo de determinar modalidades de conducta relacionadas con los ingresos y los gastos de consumo para una composición específica del hogar en términos de género. La situación de empleo se relaciona con la percepción de ingresos personales por los miembros adultos del hogar. Los hogares se clasifican de acuerdo con el número de hombres y mujeres empleados y desempleados (Grown y Valodia, 2010) y los miembros inactivos se consideran desempleados. Así, se establecen las siguientes categorías:

- Hogares con un sostén de la familia masculino: por lo menos un hombre trabaja y ninguna mujer trabaja, con y sin niños.

- Hogares con un sostén de la familia femenino: por lo menos una mujer trabaja y ningún hombre trabaja, con y sin niños.

- Hogares con dos fuentes de ingresos: por lo menos un hombre trabaja y una mujer trabaja, con y sin niños.

- Hogares donde nadie trabaja, con y sin niños.

Las primeras dos categorías se refieren a los hogares con una sola fuente de ingresos. La tercera categoría corresponde a los hogares con dos fuentes de ingresos y la última a la situación en que los miembros del hogar están desempleados. Todas las categorías se dividen en subcategorías de hogares con y sin niños (menores de 18 años). En el cuadro 2 se discriminan las participaciones en términos de cantidades de personas de cada tipo de hogar considerado.

En el cuadro 3 se muestra la distribución del ingreso para fines comparativos. Los ingresos indicados no son los originales de la encuesta de hogares sino los construidos para obtener las cifras antes de impuestos, porque los ingresos declarados de los trabajadores formales corresponden a las cifras después de impuestos. 


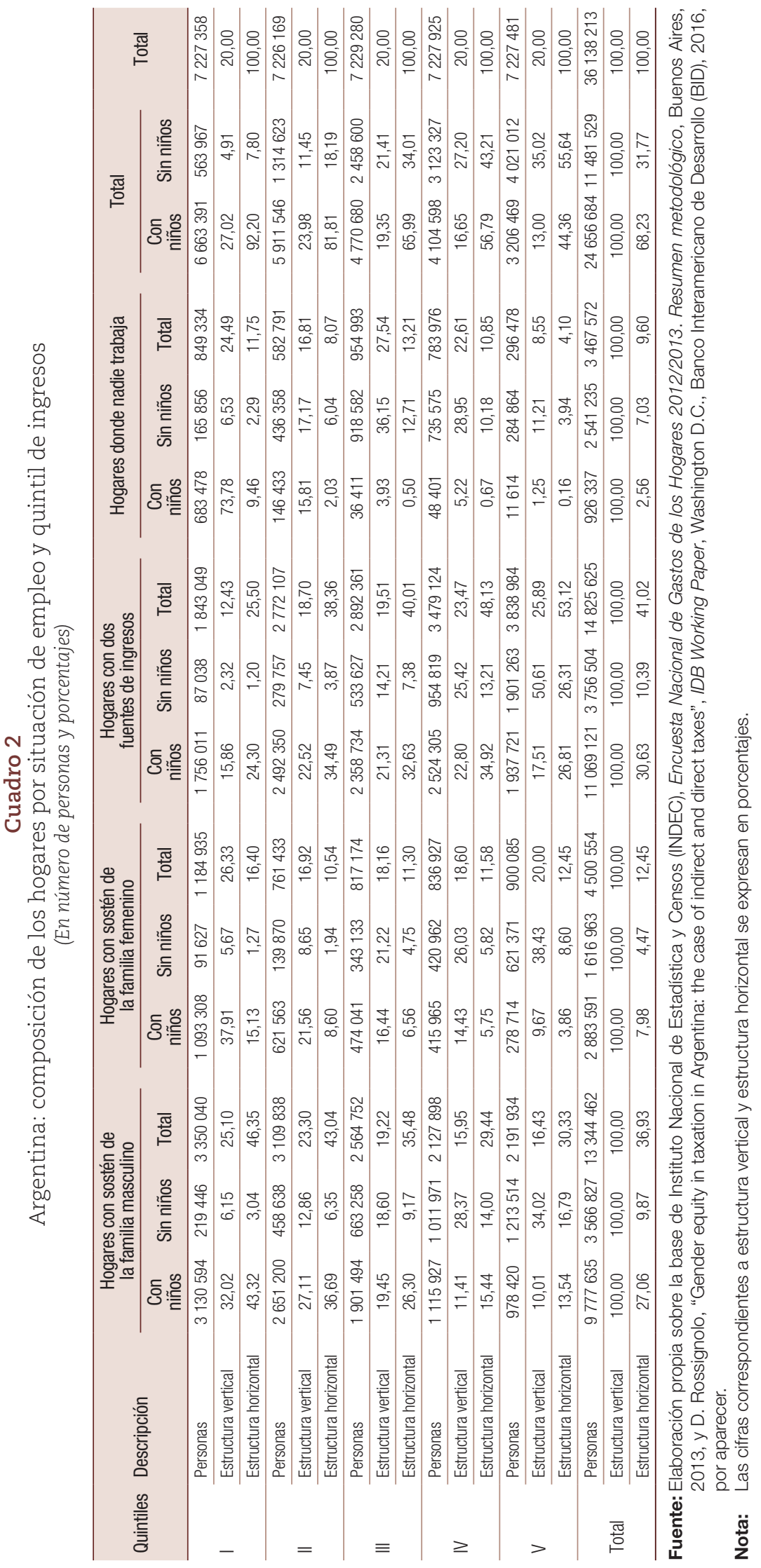




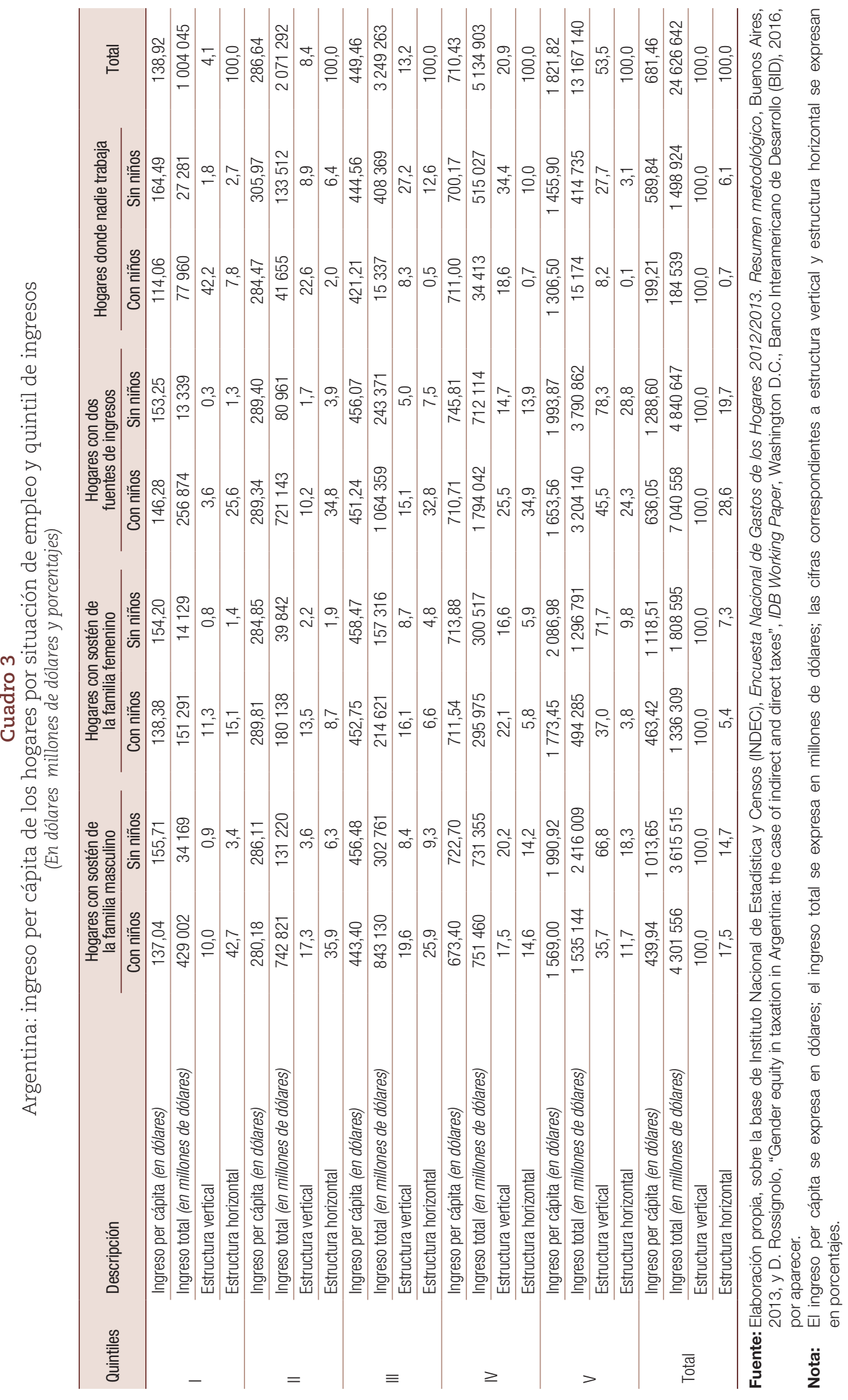


La distribución del ingreso analizada se refiere a los ingresos del hogar, según la clasificación de los hogares conforme el ingreso per cápita, agrupados en quintiles de personas. El primer quintil representa el 4,1\% de los ingresos, mientras que el quintil de mayores ingresos representa aproximadamente el 53,5\%. Los ingresos medios mensuales por hogar son de alrededor de 681 dólares corrientes (tipo de cambio de 2012). En el primer quintil, los hogares con un sostén de la familia masculino representan el $46,3 \%$ de las personas al ordenar por ingreso per cápita, mientras que los hogares con un sostén de la familia femenino comprenden el 16,4\% de las personas según el ingreso per cápita en el quintil más pobre. En los hogares con dos fuentes de ingresos se concentra el 25,5\% de las personas según la clasificación por ingresos.

En el cuadro 3 se muestra la estructura porcentual de los ingresos según el ingreso per cápita del hogar por quintiles de personas, agrupadas por fuentes de ingresos agregadas antes de impuestos. Los resultados del cuadro 3 deben combinarse con los del cuadro 2, en el que se incluía el número de personas comprendidas en cada categoría.

La menor proporción de los ingresos se concentra en los hogares con un sostén de la familia femenino y los hogares en los que nadie trabaja, categorías que representan el $16,5 \%$ y el $10,5 \%$ de los ingresos, respectivamente. Los hogares con un sostén de la familia masculino representan el 10\% (con niños) y el 0,9\% (sin niños), mientras que los hogares con dos fuentes de ingresos representan el 3,6\% (con niños) y el 0,3\% (sin niños) de los ingresos en los quintiles más bajos.

La participación en los ingresos de cada quintil aumenta considerablemente en el caso de los hogares con dos fuentes de ingresos y de los hogares con por lo menos una mujer empleada, siempre sin niños. Los primeros representan proporciones del $78 \%$ o mayores, mientras que los últimos representan proporciones del $71 \%$ o mayores.

Al comparar estas cifras con los resultados obtenidos en el cuadro 2, los hogares con un sostén de la familia femenino concentran el 12,8\% de los ingresos familiares (combinando las subcategorías con y sin niños) y el $12,4 \%$ de las personas, mientras que los hogares con un sostén de la familia masculino concentran el 32,1\% de los ingresos y el 36,9\% de las personas. En consecuencia, si bien los ingresos familiares totales son más bajos en los hogares con un sostén de la familia femenino, en términos per cápita, los hogares con un sostén de la familia masculino tienen un ingreso inferior. Al dividir las cifras correspondientes, los resultados muestran que los ingresos per cápita antes de impuestos corresponden a 435 dólares en los hogares con un sostén de la familia masculino y niños y a 1.035 dólares para los hogares sin niños. En el caso de los hogares con un sostén de la familia femenino, las cifras correspondientes son 463 y 1.118 dólares, respectivamente.

\section{Consideraciones reglamentarias y metodológicas sobre los impuestos objeto de análisis de incidencia}

En esta sección se abordan las características de los impuestos indirectos y directos analizados en este trabajo, teniendo en cuenta, en cada caso, la estructura reglamentaria del impuesto, su sistema de tarifas, la existencia de exenciones y el procedimiento metodológico utilizado. Los impuestos indirectos examinados fueron el IVA, los impuestos sobre consumos específicos, los impuestos sobre los combustibles y los impuestos provinciales sobre el volumen de negocios. Los impuestos directos analizados fueron el impuesto sobre la renta de las personas físicas, los impuestos sobre la nómina y otros impuestos mínimos sobre la renta ("monotributo"). Estos impuestos representaron alrededor del $71 \%$ de la recaudación tributaria total (niveles nacional y provincial) en 2012, mientras que las estimaciones aquí proporcionadas representan alrededor del $80 \%$ de la recaudación del universo de impuestos tomado en cuenta (asumiendo que no hay evasión fiscal). 


\section{a) Impuestos indirectos}

El IVA es un impuesto al consumo equivalente a aplicar el impuesto al valor agregado en cada fase de elaboración. Se aplica a las importaciones en forma análoga a la producción nacional, pero a las exportaciones se aplica el tipo cero (se puede recuperar mediante compensación de deudas tributarias). Entre los hechos imponibles prevalece el tipo impositivo general del $21 \%$. Las excepciones incluyen casos gravados con tipos del $27 \%$ y el 10,5\%. El primero se aplica principalmente a la facturación de servicios públicos prestados a empresas sujetas al pago de impuestos y el segundo a una lista limitada de bienes y servicios. La reglamentación relacionada con el IVA incluye una lista de exenciones ${ }^{11}$. La tendencia en el diseño de este impuesto se ha caracterizado por sucesivas reformas con miras a ampliar su base y aumentar los tipos impositivos. El consumo exento ascendió al 15\% del gasto en 2006 (Gómez Sabaini y Rossignolo, 2009).

El procedimiento para estimar la contribución al IVA de cada hogar de la muestra de la ENGHo consiste en asimilar los tipos relevantes a cada ítem de la encuesta sujeto a impuestos o exento del pago de acuerdo con la modalidad de consumo del hogar, teniendo en cuenta su transferencia a cada uno de los ítems específicos del precio del producto. Considerando que la encuesta tiene en cuenta los montos del gasto en consumo, que no se incluyen datos de precios y que el IVA es un impuesto ad valorem, si no hay otros impuestos que afecten la base imponible, el cálculo del impuesto es el siguiente:

$$
\text { taxpaid }_{i}=\text { rate }_{j} *\left(\text { expend }_{1} /\left(1+\sum_{j} \text { rate }_{j}\right)\right)
$$

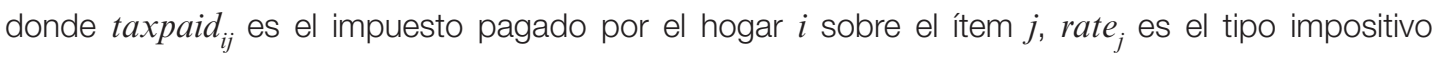
aplicado al ítem $j$ y expend $d_{i j}$ es el gasto declarado para el hogar $i$ en el ítem $j$. La incidencia tributaria para el hogar $i$ en el grupo de productos básicos $k\left(\bar{\theta}_{i}^{k}\right)$ se define como la relación entre el gasto en el impuesto (IVA) anual per cápita del hogar $i$ en el grupo de productos básicos $k\left(\bar{T}_{i}^{k}\right)$ y el ingreso per cápita del hogar $i\left(Y_{i j}\right)$ (en el caso del IVA) ${ }^{12}$

$$
\bar{\theta}_{i}^{k}=\bar{T}_{i}^{k} /\left(Y_{i_{i}}\right) \text { donde, } \bar{T}_{i}=\sum_{k} \bar{T}_{i}^{k} \text { y } \bar{T}_{i}^{k}=\sum_{j} \bar{T}_{i j}, j \in k .
$$

Los impuestos sobre consumos específicos se aplican a la venta interna y la importación de un determinado número de bienes y transacciones: tabaco, bebidas alcohólicas, cerveza, refrescos y otras bebidas no alcohólicas, automóviles y diésel. Como en todos los casos de impuestos sobre bienes, la base imponible incluye al propio impuesto. Debido a que el impuesto en sí no se puede sustraer de la base imponible, el tipo impositivo efectivo es diferente del tipo impositivo nominal. Los productos nacionales exportados están exentos. La Dirección Nacional de Investigaciones y Análisis Fiscal (DNIAF, 2013) detalla los tipos aplicados a los principales productos.

Cada uno de los impuestos sobre consumos específicos de bienes y servicios se clasificó por ítem de acuerdo con la reglamentación tributaria para establecer una correspondencia entre cada uno de ellos y un ítem similar de la ENGHo, pues se asume, como se suele hacer, que esos impuestos se transfieren completamente al precio de los productos correspondientes.

${ }^{11}$ El tipo de IVA diferencial incluye fruta fresca, refrigerada o congelada, legumbres y verduras, cereales, pan y servicios de transporte interno para pasajeros, entre otras cosas. El agua natural común, la leche sin aditivos y los medicamentos son las exenciones más importantes.

12 En el caso de los bienes exentos, se utilizó el cuadro insumo-producto de 1997, con datos de 1993. Para cada bien exento se estimó la proporción de insumo imponible, luego se estimó la incidencia de los insumos imponibles para el monto de ventas de bienes exentos y dicha estructura se aplicó al total de IVA de compras derivado del consumo de bienes exentos. 
En el caso del impuesto sobre los combustibles, a partir de 2012 se gravaron el combustible líquido y el gas natural comprimido. La DNIAF (2013) enumera los productos gravados, los tipos impositivos y las cantidades mínimas por unidad de medida. Debido a que en la actualidad no hay un estudio fiable que determine el porcentaje del costo del combustible que forma parte del costo del transporte transferido al consumidor en la Argentina y a la existencia de subvenciones al transporte y al combustible que distorsionan los valores relativos, se asumió que se transfiere el 30\% del impuesto.

El impuesto sobre el volumen de negocios provincial es muy importante en términos de recaudación para los gobiernos subnacionales y se aplica en todas las provincias. Corresponde a todas las etapas de producción y distribución de bienes y servicios y grava los ingresos brutos sin deducción de los impuestos ya pagados y acumulados mediante las compras. En los códigos tributarios de cada provincia se establecen los principios básicos del impuesto, junto con los tipos impositivos. En general, estos últimos varían del mayor al menor en el comercio y los servicios y la intermediación financiera (del 4,5\% al 5\%), la industria y el sector primario.

Para calcular la incidencia tributaria se aplicaron los tipos impositivos a los datos de consumo declarados. De acuerdo con varios autores (véase por ejemplo Rossignolo, 2015), los tipos impositivos efectivos son por lo menos dos veces más altos que los tipos sobre el consumo final. En consecuencia, los tipos al consumo al por menor se aumentaron un 150\% para explicar este efecto. Debido a que este impuesto es el más cercano a los costos de insumos, debería incluirse en los cálculos, en la base imponible del IVA y los impuestos sobre consumos específicos.

\section{b) Impuestos directos}

Por lo general, se asume que la incidencia económica del impuesto sobre la renta de las personas físicas y otros impuestos relacionados con los ingresos es igual a la incidencia legal. En el caso de los impuestos sobre la nómina, se asume que la carga recae completamente en los empleados mediante un salario reducido. En el caso de los trabajadores asalariados, las encuestas de hogares indican los ingresos netos, es decir los ingresos obtenidos después de los aportes a la seguridad social.

En las encuestas rara vez se indica la suma pagada por los encuestados por concepto de impuestos sobre la renta. En el caso de los trabajadores formales, se declaran los ingresos después de impuestos. Por consiguiente, los ingresos declarados por salarios deberían convertirse a cifras brutas para obtener los ingresos antes de impuestos. En el caso de los trabajadores asalariados informales, los empleadores, los trabajadores independientes, los perceptores de ganancias de capital, los beneficiarios de la seguridad social, los pensionistas y los beneficiarios de transferencias públicas los ingresos declarados corresponden a las sumas antes de impuestos. Para llegar a la carga fiscal, es necesario calcular la recaudación tributaria de todas esas fuentes, asumiendo que constituyen renta imponible (excepto por las transferencias).

El impuesto sobre la renta de las personas físicas es un impuesto de tipo global, estructurado con tipos impositivos progresivos, y su base imponible se ha ampliado mediante numerosas resoluciones. En la Ley del Impuesto sobre la Renta se establecen cuatro categorías de ingresos según su fuente (renta de la tierra, ganancias de capital, ingresos empresariales y de determinados agentes de negocios e ingresos personales). Un contribuyente individual puede generar ingresos correspondientes a una o más categorías al mismo tiempo. El cálculo de la renta imponible se basa en los ingresos y los gastos correspondientes a las cuatro categorías y a las participaciones en empresas o actividades. La renta imponible neta se calcula deduciendo los gastos, las deducciones personales computables, las prestaciones por familiares a cargo, el umbral de renta mínima no imponible y la 
deducción especial de los ingresos ${ }^{13}$. Los ingresos mensuales de los trabajadores asalariados están sujetos a retenciones realizadas por los empleadores, que son responsables de pagar el impuesto a las autoridades tributarias.

Las exenciones cubren, entre otras cosas, los intereses devengados por depósitos en cuentas de ahorro, depósitos a plazo, ingresos derivados de valores, acciones, bonos, letras de cambio, pagarés y otros títulos emitidos o que se han de emitir en el futuro por una autoridad gubernamental y el valor de alquiler de la vivienda cuando está ocupada por sus propietarios. Las pensiones, los pagos de jubilación y los salarios recibidos durante licencias por enfermedad no están exentos.

En el caso de los trabajadores formales, el impuesto se determina conforme la categoría de renta neta imponible, sobre la base de una escala móvil que consiste en una cantidad fija más el monto que resulta de la aplicación de un tipo que varía del 9\% al 35\% sobre el excedente de cada categoría de renta inferior (DNIAF, 2013). Para el resto de los perceptores de ingresos, los ingresos declarados corresponden a las cifras antes de impuestos, de manera que deberán aplicarse el umbral y las deducciones de acuerdo con el tramo impositivo al que corresponde la renta, con miras a calcular la recaudación tributaria.

Existe un grupo de "pequeños contribuyentes" (cuyos ingresos brutos no superan los 65.800 dólares anuales) que deben pagar impuestos en el marco de un régimen simplificado, denominado monotributo. Este consiste en un impuesto de monto fijo pagado por mes que sustituye el pago del impuesto sobre la renta de las personas físicas y el IVA por un pago único al que se suman los aportes a la seguridad social y el seguro de salud. Con arreglo a este régimen, el impuesto sobre la renta se incluye en un único pago basado en un tramo impositivo (relacionado con la facturación, el área de la superficie de las instalaciones, el uso de energía durante la producción o todos esos elementos a la vez) (DNIAF, 2013) y no se aplican normas relacionadas con la evaluación de los ingresos, deducciones, dependientes o deducciones especiales. El impuesto recaudado es un monto fijo establecido de acuerdo con la categoría de monotributo a la que pertenece el contribuyente.

Para el cálculo de la incidencia, se seleccionaron las ganancias individuales de los trabajadores independientes y los empleadores y se verificó si sus ingresos declarados (que corresponden a ingresos antes de impuestos) son inferiores al umbral establecido en el código tributario. Todos los contribuyentes seleccionados deberían excluirse de la base del impuesto sobre la renta de las personas físicas.

También se incluyeron los impuestos sobre la nómina y los aportes a la seguridad social para los trabajadores formales e informales. El cálculo se dividió en dos partes: los ingresos generados por los trabajadores formales y los montos pagados por los trabajadores independientes. Para explicar los primeros, en la encuesta de hogares se informa si el empleador retuvo en la fuente los aportes realizados por el empleado. En consecuencia, estos son quienes soportan la carga fiscal que corresponde tanto al empleador como al empleado. Para los trabajadores por cuenta propia, debido a que se asume que no hay evasión, los cálculos suponen que los trabajadores independientes realizan aportes al sistema de la seguridad social en la medida en que no hayan sido incluidos en el régimen de monotributo.

En el caso del sector formal, la estimación de incidencia se realizó sobre la base de los tipos reglamentarios (DNIAF, 2013), mediante la conversión de los ingresos netos de los trabajadores asalariados indicados en la encuesta en ingresos brutos, netos del impuesto sobre la renta de las personas físicas. En el caso de los trabajadores independientes, los tipos se aplicaron a una base imponible creciente, que difiere entre profesionales y comerciantes. Estos se identificaron en la encuesta de hogares mediante los años de educación. Al igual que en el caso de estos tipos de perceptores de ingresos, los ingresos declarados son antes de impuestos.

\footnotetext{
${ }^{13}$ Los gastos corresponden a gastos autorizados como aquellos en los que se incurre para obtener, mantener y preservar los ingresos objeto de este impuesto, que se descontarán de los ingresos producidos por la fuente que los origina.
} 


\section{Resultados de las estimaciones}

En esta sección se presentan los resultados del análisis de incidencia. La variable utilizada en el análisis es "impuesto como porcentaje de los ingresos per cápita (antes de impuestos)". En todos los cuadros se incluyen los valores medios. Los resultados se presentan por separado, considerando impuestos indirectos y directos, y según los quintiles de ingresos per cápita antes de impuestos ${ }^{14}$.

En el cuadro 4 se muestra la incidencia general del sistema tributario. Los impuestos son en general progresivos, pues la progresividad de los impuestos directos compensa con creces la regresividad de los impuestos indirectos. La carga fiscal media agregada es del 33,4\%.

Cabe observar que la carga para todos los hogares por concepto de impuestos indirectos alcanza el 14,8\% con respecto a los ingresos. La mayor parte de esa incidencia deriva del importante efecto del IVA, cuya incidencia media total es del 8,7\%. La carga fiscal agregada del impuesto sobre el volumen de negocios es del 4,5\%. La incidencia de los impuestos sobre consumos específicos y los impuestos sobre los combustibles es bastante similar, de alrededor del 1,2\% de los ingresos per cápita. La carga más alta corresponde a los hogares con un sostén de la familia femenino (16,2\%).

En conjunto, los impuestos indirectos son muy regresivos. La carga fiscal asciende al $22,4 \%$ en el primer quintil y disminuye al 8,6\% en el quintil superior. En el análisis por impuesto, el IVA resulta regresivo pues el primer quintil soporta una carga del $13,4 \%$ y el quinto quintil del $4,9 \%$. El efecto de los impuestos sobre consumos específicos también es regresivo, con porcentajes del $1,5 \%$ y el 0,3\% para el primer y el quinto quintil, respectivamente. Las diferencias son estadísticamente significativas en todos los casos. El impuesto sobre los combustibles es moderadamente proporcional, pues tanto el primer como el quinto quintil soportan una carga del 0,6\%.

En el cuadro 5 y en el gráfico 4 se presentan los resultados agregados de la incidencia estimada de los impuestos directos, indirectos y totales examinados, por tipo de hogar, mientras que en el cuadro A1.1 del anexo se presentan los resultados desglosados por tipo de impuesto. La mayor carga fiscal indirecta corresponde a los hogares donde nadie trabaja sin niños del primer quintil. La carga fiscal es más alta para los hogares con un sostén de la familia femenino con niños y la regresividad también es mayor para los hogares de esta categoría, en segundo lugar después de los hogares donde nadie trabaja sin niños. El primer quintil soporta la carga mayor $(31,8 \%)$, mientras que los hogares con dos fuentes de ingresos sin niños tienen la carga menor.

${ }^{14}$ Errores estándar disponibles mediante solicitud al autor. 


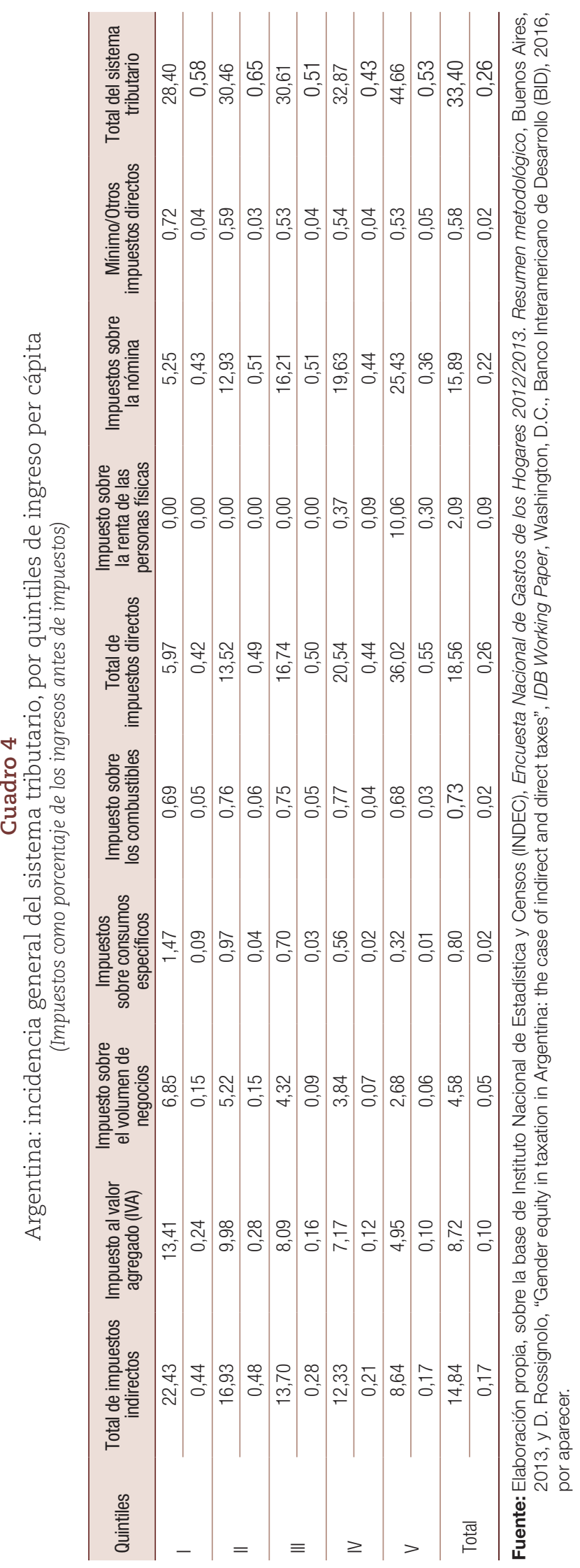




\section{Cuadro 5}

Argentina: incidencia general de los impuestos por clasificación de empleo agregada y quintiles de ingreso per cápita

(En porcentajes)

\begin{tabular}{|c|c|c|c|c|c|c|}
\hline & \multicolumn{6}{|c|}{ Quintil } \\
\hline & 1 & II & III & IV & V & Total \\
\hline & \multicolumn{6}{|c|}{ Total de impuestos directos } \\
\hline Sostén de la familia masculino CON niños & 8,07 & 19,42 & 25,78 & 26,82 & 50,52 & 20,98 \\
\hline Sostén de la familia masculino SIN niños & 6,68 & 5,57 & 8,77 & 16,13 & 30,24 & 17,62 \\
\hline Sostén de la familia masculino & 7,98 & 17,37 & 21,38 & 21,74 & 39,30 & 20,08 \\
\hline Sostén de la familia femenino CON niños & 5,66 & 10,35 & 20,21 & 25,02 & 45,43 & 15,70 \\
\hline Sostén de la familia femenino SIN niños & 1,72 & 5,22 & 8,92 & 14,04 & 29,94 & 17,60 \\
\hline Sostén de la familia femenino & 5,36 & 9,41 & 15,47 & 19,49 & 34,73 & 16,38 \\
\hline Hogares con dos fuentes de ingresos CON niños & 5,39 & 13,88 & 19,76 & 26,52 & 40,12 & 21,26 \\
\hline Hogares con dos fuentes de ingresos SIN niños & 5,11 & 6,85 & 12,94 & 19,70 & 34,05 & 24,71 \\
\hline Hogares con dos fuentes de ingresos & 5,37 & 13,17 & 18,50 & 24,65 & 37,11 & 22,13 \\
\hline Hogares donde nadie trabaja CON niños & 0,15 & 0,05 & 0,59 & 1,04 & 1,89 & 0,22 \\
\hline Hogares donde nadie trabaja SIN niños & 0,23 & 0,00 & 0,01 & 0,12 & 1,68 & 0,24 \\
\hline Hogares donde nadie trabaja & 0,17 & 0,02 & 0,03 & 0,17 & 1,69 & 0,24 \\
\hline \multirow[t]{2}{*}{ Total } & 5,97 & 13,52 & 16,74 & 20,54 & 36,02 & 18,56 \\
\hline & \multicolumn{6}{|c|}{ Total de impuestos indirectos } \\
\hline Sostén de la familia masculino CON niños & 22,13 & 15,80 & 13,32 & 12,21 & 7,94 & 16,15 \\
\hline Sostén de la familia masculino SIN niños & 23,02 & 21,24 & 15,32 & 12,90 & 9,19 & 13,78 \\
\hline Sostén de la familia masculino & 22,19 & 16,60 & 13,83 & 12,54 & 8,63 & 15,52 \\
\hline Sostén de la familia femenino CON niños & 23,19 & 19,22 & 13,13 & 13,66 & 7,69 & 17,81 \\
\hline Sostén de la familia femenino SIN niños & 22,69 & 20,29 & 15,32 & 13,42 & 9,68 & 13,50 \\
\hline Sostén de la familia femenino & 23,16 & 19,42 & 14,05 & 13,54 & 9,06 & 16,26 \\
\hline Hogares con dos fuentes de ingresos CON niños & 20,55 & 15,88 & 12,64 & 11,13 & 8,05 & 13,48 \\
\hline Hogares con dos fuentes de ingresos SIN niños & 24,90 & 19,56 & 13,83 & 12,31 & 8,32 & 11,34 \\
\hline Hogares con dos fuentes de ingresos & 20,76 & 16,25 & 12,86 & 11,46 & 8,18 & 12,93 \\
\hline Hogares donde nadie trabaja CON niños & 24,57 & 17,93 & 15,79 & 13,29 & 17,39 & 22,49 \\
\hline Hogares donde nadie trabaja SIN niños & 31,86 & 19,00 & 16,91 & 14,43 & 13,15 & 17,11 \\
\hline Hogares donde nadie trabaja & 25,99 & 18,73 & 16,87 & 14,36 & 13,32 & 18,55 \\
\hline \multirow[t]{2}{*}{ Total } & 22,43 & 16,93 & 13,87 & 12,33 & 8,64 & 14,84 \\
\hline & \multicolumn{6}{|c|}{ Total del sistema tributario } \\
\hline Sostén de la familia masculino CON niños & 30,21 & 35,22 & 39,10 & 39,04 & 58,46 & 37,13 \\
\hline Sostén de la familia masculino SIN niños & 29,70 & 26,81 & 24,09 & 29,03 & 39,43 & 31,41 \\
\hline Sostén de la familia masculino & 30,17 & 33,98 & 35,22 & 34,28 & 47,92 & 35,60 \\
\hline Sostén de la familia femenino CON niños & 28,86 & 29,57 & 33,34 & 38,68 & 53,13 & 33,51 \\
\hline Sostén de la familia femenino SIN niños & 24,41 & 25,51 & 24,24 & 27,46 & 39,61 & 31,10 \\
\hline Sostén de la familia femenino & 28,51 & 28,82 & 29,52 & 33,03 & 43,80 & 32,65 \\
\hline Hogares con dos fuentes de ingresos CON niños & 25,94 & 29,76 & 32,39 & 37,65 & 48,17 & 34,74 \\
\hline Hogares con dos fuentes de ingresos SIN niños & 30,01 & 26,42 & 26,77 & 32,01 & 42,37 & 36,04 \\
\hline Hogares con dos fuentes de ingresos & 26,13 & 29,42 & 31,36 & 36,11 & 45,29 & 35,07 \\
\hline Hogares donde nadie trabaja CON niños & 24,72 & 17,98 & 16,38 & 14,32 & 19,28 & 22,72 \\
\hline Hogares donde nadie trabaja SIN niños & 32,10 & 19,00 & 16,92 & 14,54 & 14,83 & 17,35 \\
\hline Hogares donde nadie trabaja & 26,16 & 18,75 & 16,90 & 14,53 & 15,01 & 18,78 \\
\hline Total & 28,40 & 30,46 & 30,61 & 32,87 & 44,66 & 33,40 \\
\hline
\end{tabular}

Fuente: Elaboración propia, sobre la base de Instituto Nacional de Estadística y Censos (INDEC), Encuesta Nacional de Gastos de los Hogares 2012/2013. Resumen metodológico, Buenos Aires, 2013, y D. Rossignolo, "Gender equity in taxation in Argentina: the case of indirect and direct taxes", IDB Working Paper, Washington, D.C., Banco Interamericano de Desarrollo (BID), 2016, por aparecer. 


\section{Gráfico 4}

Argentina: incidencia de los impuestos indirectos por clasificación de empleo agregada y quintiles de ingreso per cápita

(Impuestos como porcentaje de los ingresos antes de impuestos)

\section{A. Impuestos indirectos}

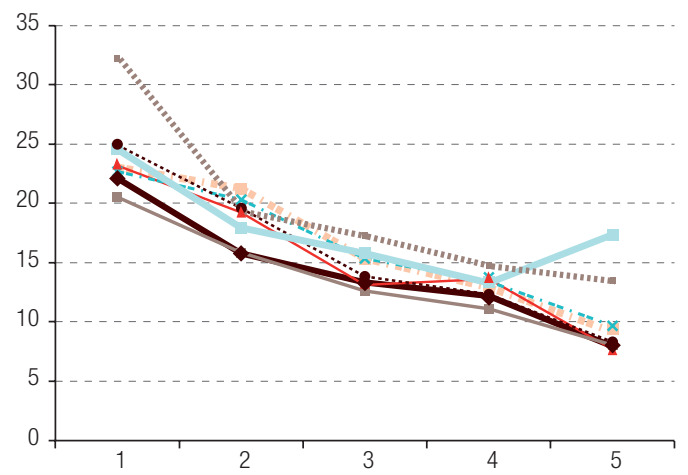

C. Impuesto sobre consumos específicos

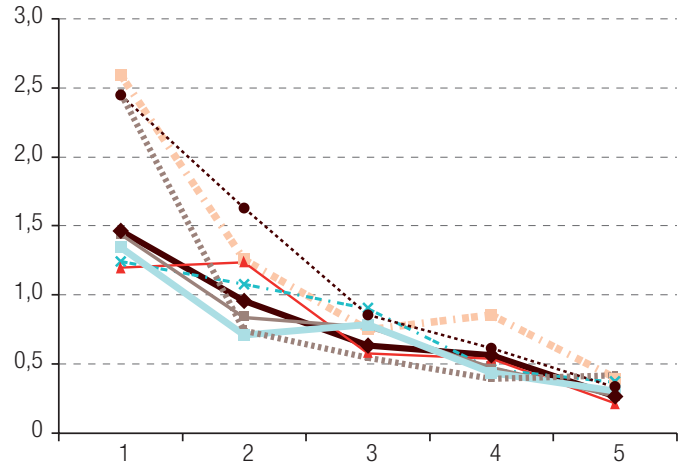

B. Impuesto al valor agregado (IVA)

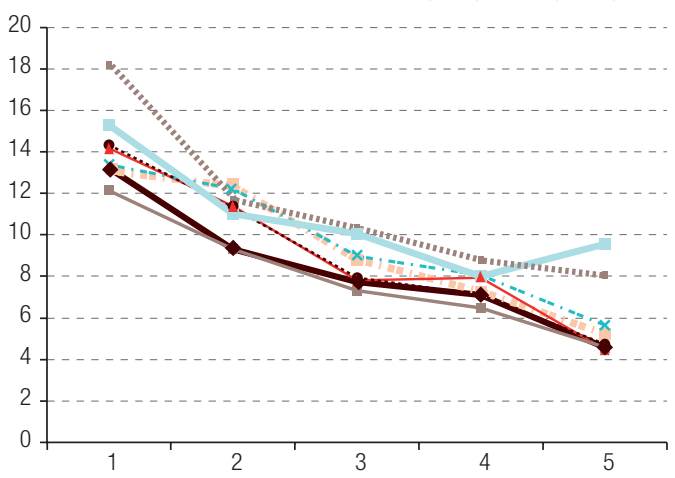

D. Impuesto sobre los combustibles

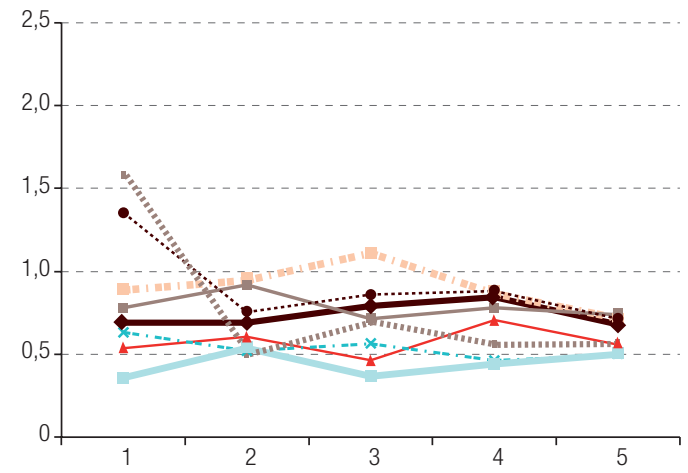

— Sostén de la familia masculino CON niños

$\rightarrow$ Hogares con dos fuentes de ingresos CON niños

$-\rightarrow x$ - Sostén de la familia femenino SIN niños

- Sostén de la familia masculino SIN niños

$\longleftarrow$ Sostén de la familia femenino CON niños

-- Hogares con dos fuentes de ingresos SIN niños

- Hogares donde nadie trabaja CON niños

" $=$ " Hogares donde nadie trabaja SIN niños

Fuente: Elaboración propia, sobre la base de Instituto Nacional de Estadística y Censos (INDEC), Encuesta Nacional de Gastos de los Hogares 2012/2013. Resumen metodológico, Buenos Aires, 2013, y D. Rossignolo, "Gender equity in taxation in Argentina: the case of indirect and direct taxes", IDB Working Paper, Washington, D.C., Banco Interamericano de Desarrollo (BID), 2016, por aparecer.

La incidencia del IVA por tipo de hogar y quintil es la que más influye en el comportamiento del impuesto general. La incidencia de este impuesto se vuelve marcadamente alta, especialmente en los segmentos inferiores de la distribución. Si bien los hogares en los que nadie trabaja sin niños del quintil más pobre enfrentan la mayor carga por concepto de IVA, los hogares en donde las mujeres son perceptoras de renta siguen soportando una carga mayor con respecto a los hogares encabezados por hombres y a los hogares con dos fuentes de ingresos en el primer quintil.

Los hogares con un sostén de la familia masculino sin niños presentan la mayor regresividad en el primer quintil, pero esto se compensa en el segundo quintil por los hogares con dos fuentes de ingresos para los impuestos sobre consumos específicos. En el cuarto y el quinto quintil, los hogares con un sostén de la familia masculino ocupan nuevamente el primer lugar con respecto a la carga tributaria.

El impuesto sobre los combustibles es moderadamente proporcional. La mayor carga en el primer quintil corresponde a los hogares con dos fuentes de ingresos y a aquellos en los que nadie 
trabaja, mientras que en el caso del impuesto sobre el volumen de negocios, el patrón muestra regresividad excepto para los hogares en los que nadie trabaja con niños, que presentan un patrón en forma de $U$. También sostienen la mayor carga por concepto de impuesto sobre los combustibles.

En el caso de los impuestos directos, se incluyeron en las estimaciones el impuesto sobre la renta de las personas físicas, los impuestos sobre la nómina y los impuestos mínimos o presuntos sobre la renta. Al evaluar los resultados se debe tener en cuenta que la importancia del impuesto sobre la renta de las personas físicas es menor que en los países de la Organización para la Cooperación y el Desarrollo Económicos (OCDE) (Gómez Sabaini, Jiménez y Rossignolo, 2012), lo que reduce el efecto redistributivo del impuesto. Esto determina que el impuesto sobre la renta de las personas físicas esté muy concentrado en los quintiles de ingreso más altos, de manera que los tramos impositivos más bajos quedan excluidos de la red del impuesto. Estas personas, y muchas de las incluidas en los quintiles de ingresos más altos pero que trabajan por cuenta propia, deben pagar el impuesto mínimo o presunto (monotributo), cuya distribución de la carga fiscal es objeto de debate.

En el cuadro 4 se puede observar que la carga para todos los hogares e impuestos llega al 18,5\%. La mayor parte de esa incidencia deriva del importante efecto de los impuestos sobre la nómina, cuya incidencia media total es del 15,8\%. En conjunto, los impuestos directos son en general marcadamente progresivos. La carga corresponde al 5,9\% en el primer quintil, es decir los hogares con menores ingresos, y aumenta al 36\% en el quintil superior. Sin embargo, de acuerdo con el análisis por impuesto, el impuesto sobre la renta de las personas físicas y los impuestos sobre la nómina son progresivos, mientras que el monotributo es moderadamente regresivo (el primer quintil soporta una carga del $0,7 \%$ y el quinto del $0,5 \%$ ). Todas las diferencias en las cargas fiscales entre quintiles y dentro de las distintas categorías de hogares son estadísticamente significativas (véanse el cuadro 5 y el cuadro A1.1 del anexo) ${ }^{15}$.

En el gráfico 5 se muestran los resultados agregados de la incidencia estimada de los impuestos directos examinados, por tipo de hogar. La carga más alta corresponde a los hogares con dos fuentes de ingresos (22,1\%). Todos los demás impuestos presentan un patrón similar, con excepción del monotributo. Casi todos los tipos de hogares presentan un patrón progresivo similar para los primeros cuatro quintiles, con un valor máximo en el quinto debido al impuesto sobre la renta de las personas físicas. La carga más alta corresponde a los hogares con un sostén de la familia masculino en todos los quintiles de ingreso. En todos los casos, la carga tributaria es mayor para los hogares con niños.

El impuesto sobre la renta de las personas físicas recae casi completamente en los hogares comprendidos en el cuarto y el quinto quintil. Los hogares con niños soportan la carga más elevada de este impuesto, debido a que este tipo de hogares concentra una mayor proporción de ingresos que los hogares sin niños y a que los hogares con un sostén de la familia masculino son los que presentan la carga más alta en el quinto quintil.

Es evidente que, debido a los umbrales y las deducciones, pocas personas pagan el impuesto sobre la renta de las personas físicas. La incidencia de este impuesto se vuelve progresiva y se concentra en el quintil más alto. La carga más alta recae en los hogares con un sostén de la familia masculino y los hogares con un sostén de la familia femenino. Los hogares en los que nadie trabaja presentan la carga más baja de este impuesto, debido a que se encuentran fuera del sector formal.

La incidencia de los impuestos sobre la nómina también es progresiva cuando se calcula sobre los ingresos antes de impuestos y, una vez más, la carga mayor corresponde a los hogares con un sostén de la familia masculino con niños. Por el contrario, el monotributo es moderadamente regresivo. La carga más alta recae en los hogares con un sostén de la familia masculino sin niños, mientras que este impuesto es más regresivo para los hogares con dos fuentes de ingresos sin niños.

\footnotetext{
${ }^{15}$ Todas las pruebas de significación entre las distintas categorías de hogares y entre los diferentes quintiles dentro de cada categoría de hogares están disponibles mediante solicitud al autor.
} 


\section{Gráfico 5}

Argentina: incidencia de los impuestos directos y totales por clasificación de empleo agregada y quintiles de ingreso per cápita (Impuestos como porcentaje de los ingresos antes de impuestos)

\section{A. Impuestos directos}

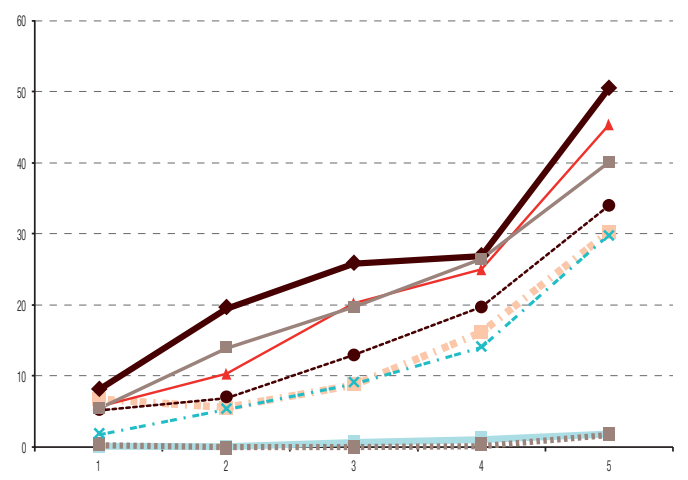

C. Impuestos sobre la nómina

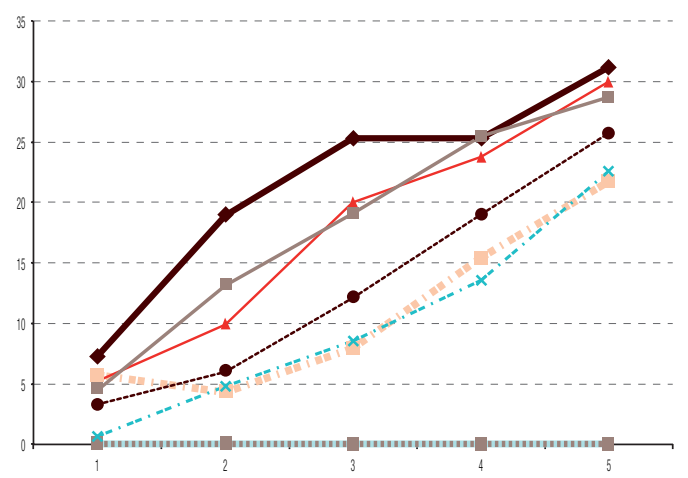

B. Impuesto sobre la renta de las personas físicas

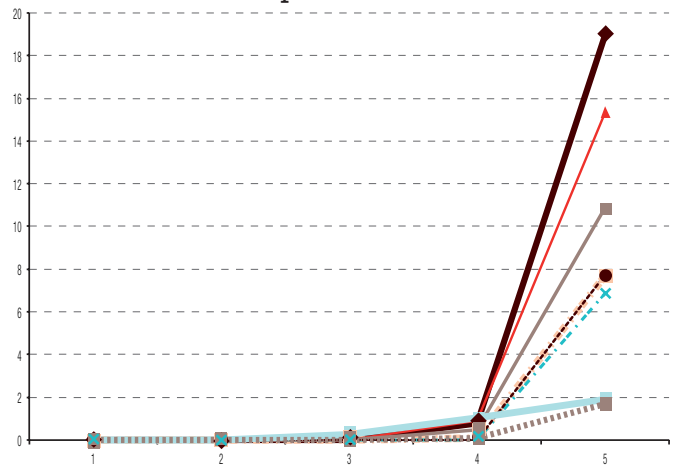

D. Impuestos totales

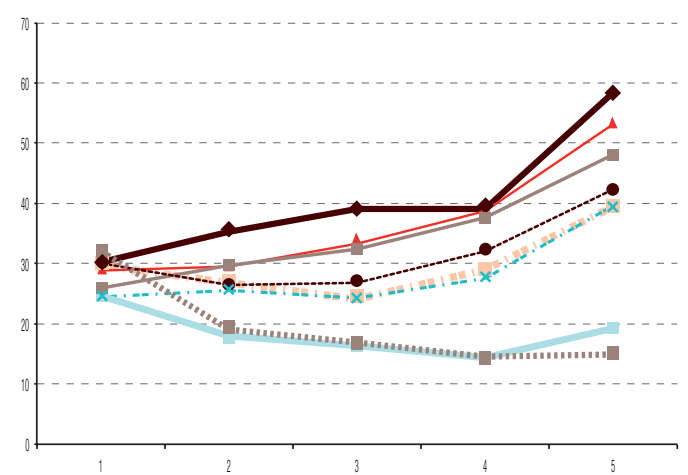

— Sostén de la familia masculino CON niños - Hogares con dos fuentes de ingresos CON niños $--\star$ - Sostén de la familia femenino SIN niños
- Sostén de la familia masculino SIN niñoS

-.- Hogares con dos fuentes de ingresos SIN niños "म" Hogares donde nadie trabaja SIN niños
— Sostén de la familia femenino CON niños Hogares donde nadie trabaja CON niños

Fuente: Elaboración propia, sobre la base de Instituto Nacional de Estadística y Censos (INDEC), Encuesta Nacional de Gastos de los Hogares 2012/2013. Resumen metodológico, Buenos Aires, 2013, y D. Rossignolo, "Gender equity in taxation in Argentina: the case of indirect and direct taxes", IDB Working Paper, Washington, D.C., Banco Interamericano de Desarrollo (BID), 2016, por aparecer.

En el caso del impuesto sobre la renta de las personas físicas, por ejemplo, si bien la carga media más alta corresponde a los hogares con un sostén de la familia masculino sin niños (2,6\%), en el quinto quintil el impuesto más alto es pagado por los hogares con un sostén de la familia masculino con niños. En el caso del monotributo, los hogares con un sostén de la familia masculino con niños presentan la carga más elevada, que incluso aumenta para el quintil más rico. Los hogares en donde las mujeres son perceptoras de ingresos presentan una carga mayor que aquellos encabezados por hombres en el primer quintil (comprenden más trabajos independientes que quedan fuera del impuesto sobre la renta de las personas físicas).

En conjunto, el sistema tributario general (en la medida de los impuestos analizados) es progresivo. La carga tributaria recae mayormente en los hogares con un sostén de la familia masculino, con un sostén de la familia femenino y con dos fuentes de ingresos, y aumenta para todos los tipos de hogares con niños (excepto en el caso de aquellos en los que nadie trabaja). La carga para los 
hogares sin niños tiende a ser más proporcional, mientras que la carga para los hogares en los que nadie trabaja es más regresiva. Como ya se mencionó, estos resultados se basan en que, en términos per cápita, los hogares con un sostén de la familia masculino tienen un ingreso inferior al de los hogares con un sostén de la familia femenino.

\section{Resumen y conclusiones}

El principal objetivo de esta investigación era analizar la manera en que los sistemas tributarios afectan la equidad de género en la Argentina. La pregunta clave detrás de la observación era si el sistema tributario promueve una mayor equidad de género. En las últimas dos décadas, el sistema tributario argentino ha sido objeto de considerables y frecuentes cambios, que no formaban parte de una reforma integral y, por el contrario, estaban diseñados como medidas provisionales, con miras a generar recursos para cubrir déficits presupuestarios. Muy pocas de esas reformas tenían el objetivo de incrementar la equidad.

El análisis de los impuestos indirectos incluyó el IVA, los impuestos sobre consumos específicos, el impuesto sobre los combustibles y los impuestos provinciales sobre el volumen de negocios. Los impuestos directos incluyeron el impuesto sobre la renta de las personas físicas, los impuestos sobre la nómina y los impuestos mínimos o presuntos. El indicador de bienestar empleado fueron los ingresos per cápita antes de impuestos y el análisis se realizó por quintiles de distribución del ingreso per cápita y categorías de empleo, que se utilizaron también para clasificar a las personas.

Al analizar la carga tributaria de los impuestos indirectos sobre los ingresos surgen diferencias notables. Por ejemplo, los hogares en donde las mujeres son perceptoras de renta soportan la carga más elevada, pues estos se encuentran más concentrados en la distribución inferior del ingreso que otros tipos de hogares, como los encabezados por hombres y los que tienen dos fuentes de ingresos. Se observa una gran regresividad en el sistema tributario indirecto en general, debido al IVA, los impuestos sobre consumos específicos y el impuesto sobre el volumen de negocios. Esta característica no puede compensarse mediante el impuesto sobre los combustibles que, al contrario de los otros casos, es moderadamente proporcional. A los hogares sin niños corresponde una mayor proporción de la carga fiscal y las categorías de hogares encabezados por mujeres son las que en general soportan la mayor parte de los impuestos.

La tributación sobre la renta de las personas físicas en la Argentina presenta puntos de fortaleza y debilidad en términos de equidad de género. El principio de tributación individual es una de las fortalezas del sistema porque no desalienta a priori la generación de ingresos por las mujeres. Sin embargo, existe una discriminación formal en contra de las mujeres al no reconocerse su cuota líquida por "ingresos en común".

Un aspecto importante del sistema del impuesto sobre la renta de las personas físicas de la Argentina es su segmentación en tres categorías de contribución diferentes (trabajadores asalariados, trabajadores por cuenta propia de altos ingresos y trabajadores por cuenta propia de bajos ingresos o "monotributistas") y el tratamiento diferenciado que reciben con respecto a umbrales y deducciones. Existe otra discriminación que surge del código del impuesto sobre la renta de las personas físicas en la Argentina, que supone al mismo tiempo inequidad horizontal y vertical: el caso de las exenciones. En la Argentina, las personas que obtienen sus ingresos de su trabajo personal deben pagar el impuesto sobre la renta (siempre que sus ingresos superen el umbral). Por el contrario, las personas que obtienen sus ingresos de ganancias financieras (por ejemplo, mediante la participación en el mercado bursátil) no deben hacerlo. Cabe señalar que dentro de este último grupo es más probable encontrar personas de mayores ingresos y en particular hombres. 
Los hogares con niños soportan la mayor carga fiscal directa, sobre todo aquellos con un sostén de la familia masculino o dos fuentes de ingresos. El efecto limitado del impuesto sobre la renta de las personas físicas - pagado solo por aproximadamente el 30\% de la población en la categoría superior de la clasificación por ingreso per cápita - reduce el alcance de este impuesto para promover políticas orientadas a la equidad.

Para reducir la carga de los impuestos indirectos en los hogares más pobres en donde las mujeres son perceptoras de renta se sugiere una serie de medidas de política. Por ejemplo, la reducción del IVA sobre una canasta de alimentos seleccionados (que incluya también transporte público y prendas de vestir para niños) y el incremento de los impuestos sobre los artículos de lujo aumentarían los efectos de equidad de los impuestos indirectos. En el caso de los impuestos directos, la ampliación de la base imponible del impuesto sobre la renta de las personas físicas (es decir, la tributación de las ganancias de capital, que se concentran principalmente en los hogares encabezados por hombres) también mejoraría la equidad de género.

\section{Bibliografía}

Bourguignon, F. y L. Pereira da Silva (2003), "Evaluating the poverty and distributional impact of economic policies: a compendium of existing techniques", The Impact of Economic Policies on Poverty and Income Distribution: Evaluation Techniques and Tools, F. Bourguignon y L. Pereira da Silva (eds.), Washington, D.C., Banco Mundial.

DNIAF (Dirección Nacional de Investigaciones y Análisis Fiscal) (2013), "Tributos vigentes en la República Argentina 2012", Buenos Aires.

Doss, C. (2011), "Intrahousehold bargaining and resource allocation in developing countries" [en línea] http:// siteresources. worldbank.org/INTWDR2012/Resources/7778105-12996999685583/7786210-1322671773271/ Doss-Intrahh-bargaining.pdf.

Gasparini, L. (1998), "Incidencia distributiva del sistema impositivo argentino", La reforma tributaria en la Argentina, Buenos Aires, Fundación de Investigaciones Económicas Latinoamericanas (FIEL).

Gómez Sabaini, J. C., M. Harriague y D. Rossignolo (2013), "La situación fiscal en Argentina y sus efectos sobre la distribución del ingreso", Desarrollo Económico, vol. 52, № 207-208, Buenos Aires, Instituto de Desarrollo Económico y Social (IDES).

Gómez Sabaini, J. C., J. P. Jiménez y D. Rossignolo (2012), "Imposición a la renta personal y equidad en América Latina: nuevos desafíos", serie Macroeconomía del Desarrollo, №119 (LC/L.3477-P), Santiago, Comisión Económica para América Latina y el Caribe (CEPAL).

Gómez Sabaini, J. C. y D. Rossignolo (2009), "Argentina: análisis de la situación tributaria y propuestas de reformas impositivas destinadas a mejorar la distribución del ingreso", Reflexiones y propuestas para mejorar la distribución del ingreso en Argentina, S. Keifman, Buenos Aires, Organización Internacional del Trabajo (OIT).

Gómez Sabaini, J. C., J. J. Santiere y D. Rossignolo (2002), "La equidad distributiva y el sistema tributario: un análisis para el caso argentino", serie Gestión Pública, № 20 (LC/L.1733-P), Santiago, Comisión Económica para América Latina y el Caribe (CEPAL).

Grown, C. (2010), "Taxation and gender equality", Taxation and Gender Equity: A Comparative Analysis of Direct and Indirect Taxes in Developing and Developed Countries, C. Grown e I. Valodia (eds.), Nueva York, Routledge.

Grown, C. e I. Valodia (eds.) (2010), Taxation and Gender Equity: A Comparative Analysis of Direct and Indirect Taxes in Developing and Developed Countries, C. Grown e I. Valodia (eds.), Nueva York, Routledge.

INDEC (Instituto Nacional de Estadística y Censos) (2013), Encuesta Nacional de Gastos de los Hogares 2012/2013. Resumen metodológico, Buenos Aires.

Lambert, P. (1993), The Distribution and Redistribution of Income: A Mathematical Analysis, Manchester, Manchester University Press.

Rodríguez Enríquez, C., N. Gherardi y D. Rossignolo (2010), "Gender equality and taxation: the Argentine case", Taxation and Gender Equity: A Comparative Analysis of Direct and Indirect Taxes in Developing and Developed Countries, C. Grown e I. Valodia (eds.), Nueva York, Routledge. 
Rossignolo, D. (2016), "Gender equity in taxation in Argentina: the case of indirect and direct taxes", IDB Working Paper, Washington, D.C., Banco Interamericano de Desarrollo (BID), por aparecer.

_ (2015), "Externalidades tributarias verticales: una estimación empírica en impuestos sobre los consumos en Argentina", Revista de Economía Pública Urbana, № 21, Santiago de Compostela, Universidad de Santiago de Compostela.

Sahn, D. y S. Younger (2003), "Estimating the incidence of indirect taxes in developing countries", The Impact of Economic Policies on Poverty and Income Distribution: Evaluation Techniques and Tools, F. Bourguignon y L. Pereira da Silva (eds.), Washington, D.C., Banco Mundial.

Stotsky, J. (1996), "Gender bias in tax systems", IMF Working Paper, № 96/99, Washington, D.C., Fondo Monetario Internacional (FMI). 


\section{Anexo A1}

\section{Cuadro A1.1}

Argentina: incidencia de los impuestos por tipo de impuesto, clasificación de empleo agregada y quintiles de ingreso per cápita

(Impuestos como porcentaje de los ingresos antes de impuestos)

\begin{tabular}{|c|c|c|c|c|c|c|c|c|c|c|c|c|}
\hline & \multicolumn{6}{|c|}{ Quintil } & \multicolumn{6}{|c|}{ Quintil } \\
\hline & 1 & $\|$ & III & IV & V & Total & 1 & II & III & IV & V & Total \\
\hline & \multicolumn{6}{|c|}{ Impuesto sobre la renta de las personas físicas } & \multicolumn{6}{|c|}{ Impuesto sobre el volumen de negocios } \\
\hline Sostén de la familia masculino CON niños & 0,00 & 0,00 & 0,00 & 0,78 & 18,94 & 1,98 & 6,77 & 4,82 & 4,16 & 3,72 & 2,41 & 4,95 \\
\hline Sostén de la familia masculino SIN niños & 0,00 & 0,00 & 0,00 & 0,10 & 7,69 & 2,65 & 6,45 & 6,69 & 4,64 & 3,91 & 2,84 & 4,20 \\
\hline Sostén de la familia masculino & 0,00 & 0,00 & 0,00 & 0,46 & 12,71 & 2,16 & 6,75 & 5,10 & 4,28 & 3,81 & 2,65 & 4,75 \\
\hline Sostén de la familia femenino CON niños & 0,00 & 0,00 & 0,01 & 0,93 & 15,33 & 1,62 & 7,30 & 5,98 & 4,28 & 4,48 & 2,44 & 5,64 \\
\hline Sostén de la familia femenino SIN niños & 0,00 & 0,00 & 0,00 & 0,01 & 6,88 & 2,65 & 7,44 & 6,46 & 4,87 & 4,44 & 3,17 & 4,39 \\
\hline Sostén de la familia femenino & 0,00 & 0,00 & 0,00 & 0,47 & 9,50 & 1,99 & 7,31 & 6,07 & 4,53 & 4,46 & 2,94 & 5,19 \\
\hline $\begin{array}{l}\text { Hogares con dos fuentes de } \\
\text { ingresos CON niños }\end{array}$ & 0,00 & 0,00 & 0,00 & 0,46 & 10,88 & 2,01 & 6,18 & 4,82 & 3,85 & 3,41 & 2,45 & 4,10 \\
\hline $\begin{array}{l}\text { Hogares con dos fuentes de } \\
\text { ingresos SIN niños }\end{array}$ & 0,00 & 0,00 & 0,00 & 0,01 & 7,74 & 3,92 & 6,77 & 5,88 & 4,16 & 3,70 & 2,55 & 3,42 \\
\hline Hogares con dos fuentes de ingresos & 0,00 & 0,00 & 0,00 & 0,34 & 9,33 & 2,49 & 6,21 & 4,93 & 3,91 & 3,49 & 2,50 & 3,92 \\
\hline Hogares donde nadie trabaja CON niños & 0,00 & 0,00 & 0,29 & 1,04 & 1,89 & 0,09 & 7,60 & 5,68 & 4,57 & 4,41 & 7,04 & 7,00 \\
\hline Hogares donde nadie trabaja SIN niños & 0,00 & 0,00 & 0,00 & 0,10 & 1,68 & 0,22 & 9,86 & 6,29 & 5,56 & 4,88 & 4,33 & 5,63 \\
\hline Hogares donde nadie $t$ & 0,00 & 0,00 & 0,01 & 0,16 & 1,69 & 0,18 & 8,04 & 6,13 & 5,52 & 4,85 & 4,43 & 6,00 \\
\hline \multirow[t]{2}{*}{ Total } & 0,00 & 0,00 & 0,00 & 0,37 & 10,06 & 2,09 & 6,85 & 5,22 & 4,32 & 3,84 & 2,68 & 4,58 \\
\hline & \multicolumn{6}{|c|}{ Impuestos sobre la nómina } & \multicolumn{6}{|c|}{ Impuestos sobre consumos específicos } \\
\hline Sostén de la familia masculino & 7,28 & 18,91 & 25,27 & 25,31 & 31,21 & 18,38 & 1,46 & 0,96 & 0,63 & 0,56 & 0,27 & 0,94 \\
\hline Sostén de la familia masculino SIN niños & 5,71 & 4,30 & 7,99 & 15,43 & 21,71 & 14,15 & 2,59 & 1,26 & 0,75 & 0,86 & 0,40 & 0,84 \\
\hline Sostén de la familia masculino & 7,18 & 16,76 & 20,80 & 20,61 & 25,95 & 17,25 & 1,54 & 1,00 & 0,66 & 0,70 & 0,34 & 0,92 \\
\hline Sostén de la familia femenino CON niños & 5,23 & 9,95 & 20,02 & 23,77 & 29,97 & 13,74 & 1,20 & 1,24 & 0,58 & 0,54 & 0,21 & 0,91 \\
\hline Sostén de la familia femenino SIN n & 0,66 & 4,78 & 8,55 & 13,60 & 22,57 & 14,48 & 1,24 & 1,08 & 0,90 & 0,46 & 0,37 & 0,62 \\
\hline Sostén de la familia femenino & 4,88 & 9,00 & 15,20 & 18,66 & 24,86 & 14,01 & 1,20 & 1,21 & 0,71 & 0,50 & 0,32 & 0,81 \\
\hline $\begin{array}{l}\text { Hogares con dos fuentes de } \\
\text { ingresos CON niños }\end{array}$ & 4,46 & 13,17 & 19,06 & 25,48 & 28,68 & 18,56 & 1,44 & 0,84 & 0,77 & 0,48 & 0,27 & 0,74 \\
\hline $\begin{array}{l}\text { Hogares con dos fuentes de } \\
\text { ingresos SIN niños }\end{array}$ & 3,31 & 5,98 & 12,10 & 19,00 & 25,76 & 20,11 & 2,45 & 1,63 & 0,86 & 0,62 & 0,33 & 0,62 \\
\hline Hogares con dos fuentes de ingresos & 4,41 & 12,44 & 17,77 & 23,70 & 27,23 & 18,95 & 1,49 & 0,92 & 0,78 & 0,51 & 0,30 & 0,71 \\
\hline Hogares donde nadie trabaja CON niños & 0,00 & 0,00 & 0,00 & 0,00 & 0,00 & 0,00 & 1,34 & 0,71 & 0,79 & 0,44 & 0,31 & 1,16 \\
\hline Hogares donde nadie trabaja SIN niños & 0,00 & 0,00 & 0,00 & 0,00 & 0,00 & 0,00 & 2,46 & 0,75 & 0,54 & 0,39 & 0,42 & 0,65 \\
\hline Hogares donde nadie trabaja & 0,00 & 0,00 & 0,00 & 0,00 & 0,00 & 0,00 & 1,56 & 0,74 & 0,55 & 0,40 & 0,41 & 0,78 \\
\hline \multirow[t]{2}{*}{ Total } & 5,25 & 12,93 & 16,21 & 19,63 & 25,43 & 15,89 & 1,47 & 0,97 & 0,70 & 0,56 & 0,32 & 0,80 \\
\hline & \multicolumn{6}{|c|}{ Mínimo/Otros impuestos directos } & \multicolumn{6}{|c|}{ Impuesto sobre los combustibles } \\
\hline Sostén de la familia masculino CON niños & 0,79 & 0,50 & 0,51 & 0,74 & 0,37 & 0,61 & 0,69 & 0,69 & 0,79 & 0,84 & 0,68 & 0,73 \\
\hline Sostén de la familia masculino SIN niños & 0,97 & 1,27 & 0,78 & 0,60 & 0,84 & 0,82 & 0,89 & 0,95 & 1,11 & 0,87 & 0,72 & 0,87 \\
\hline Sostén de la familia masculino & 0,80 & 0,62 & 0,58 & 0,67 & 0,63 & 0,67 & 0,71 & 0,73 & 0,87 & 0,85 & 0,70 & 0,77 \\
\hline Sostén de la familia femenino CON niños & 0,43 & 0,40 & 0,19 & 0,32 & 0,14 & 0,34 & 0,54 & 0,61 & 0,46 & 0,71 & 0,56 & 0,57 \\
\hline Sostén de la familia femenino SIN niños & 1,06 & 0,44 & 0,37 & 0,42 & 0,48 & 0,47 & 0,63 & 0,52 & 0,56 & 0,46 & 0,49 & 0,51 \\
\hline Sostén de la familia femenino & 0,48 & 0,41 & 0,26 & 0,37 & 0,38 & 0,39 & 0,54 & 0,59 & 0,51 & 0,58 & 0,51 & 0,55 \\
\hline $\begin{array}{l}\text { Hogares con dos fuentes de } \\
\text { ingresos CON niños }\end{array}$ & 0,93 & 0,72 & 0,70 & 0,58 & 0,55 & 0,69 & 0,78 & 0,92 & 0,71 & 0,78 & 0,74 & 0,79 \\
\hline $\begin{array}{l}\text { Hogares con dos fuentes de } \\
\text { ingresos SIN niños }\end{array}$ & 1,80 & 0,87 & 0,84 & 0,70 & 0,55 & 0,68 & 1,35 & 0,76 & 0,86 & 0,88 & 0,71 & 0,79 \\
\hline Hogares con dos fuentes de ingresos & 0,97 & 0,73 & 0,73 & 0,61 & 0,55 & 0,68 & 0,81 & 0,90 & 0,74 & 0,81 & 0,73 & 0,79 \\
\hline Hogares donde nadie trabaja CON niños & 0,15 & 0,05 & 0,30 & 0,00 & 0,00 & 0,13 & 0,36 & 0,54 & 0,37 & 0,44 & 0,50 & 0,39 \\
\hline Hogares donde nadie trabaja SIN niños & 0,23 & 0,00 & 0,01 & 0,01 & 0,00 & 0,02 & 1,56 & 0,48 & 0,67 & 0,54 & 0,54 & 0,64 \\
\hline Hogares donde nadie trabaja & 0,17 & 0,02 & 0,02 & 0,01 & 0,00 & 0,05 & 0,59 & 0,49 & 0,66 & 0,53 & 0,54 & 0,58 \\
\hline Total & 0,72 & 0,59 & 0,53 & 0,54 & 0,53 & 0,58 & 0,69 & 0,76 & 0,75 & 0,77 & 0,68 & 0,73 \\
\hline
\end{tabular}


Cuadro A1.1 (conclusión)

\begin{tabular}{|c|c|c|c|c|c|c|c|c|c|c|c|c|}
\hline & \multicolumn{6}{|c|}{ Quintil } & \multicolumn{6}{|c|}{ Quintil } \\
\hline & I & ॥ & III & IV & V & Total & 1 & $\|$ & IIII & IV & V & Total \\
\hline & \multicolumn{6}{|c|}{ Impuesto al valor agregado (IVA) } & \multicolumn{6}{|c|}{ Total del sistema tributario } \\
\hline Sostén de la familia masculino CON niños & 13,21 & 9,33 & 7,74 & 7,09 & 4,58 & 9,53 & 30,21 & 35,22 & 39,10 & 39,04 & 58,46 & 37,13 \\
\hline Sostén de la familia masculino SIN niños & 13,09 & 12,34 & 8,82 & 7,26 & 5,23 & 7,87 & 29,70 & 26,81 & 24,09 & 29,03 & 39,43 & 31,41 \\
\hline Sostén de la familia masculino & 13,20 & 9,77 & 8,02 & 7,17 & 4,94 & 9,09 & 30,17 & 33,98 & 35,22 & 34,28 & 47,92 & 35,60 \\
\hline Sostén de la familia femenino CON niños & 14,16 & 11,40 & 7,82 & 7,94 & 4,49 & 10,69 & 28,86 & 29,57 & 33,34 & 38,68 & 53,13 & 33,51 \\
\hline Sostén de la familia femenino $\mathrm{S}$ & 13,38 & 12,23 & 8,98 & 8,06 & 5,65 & 7,99 & 24,41 & 25,51 & 24,24 & 27,46 & 39,61 & 31,10 \\
\hline Sostén de la familia femenino & 14,10 & 11,55 & 8,31 & 8,00 & 5,29 & 9,72 & 28,51 & 28,82 & 29,52 & 33,03 & 43,80 & 32,65 \\
\hline $\begin{array}{l}\text { Hogares con dos fuentes de } \\
\text { ingresos CON niños }\end{array}$ & 12,15 & 9,30 & 7,30 & 6,46 & 4,59 & 7,85 & 25,94 & 29,76 & 32,39 & 37,65 & 48,17 & 34,74 \\
\hline $\begin{array}{l}\text { Hogares con dos fuentes de } \\
\text { ingresos SIN niños }\end{array}$ & 14,33 & 11,30 & 7,95 & 7,11 & 4,72 & 6,50 & 30,01 & 26,42 & 26,77 & 32,01 & 42,37 & 36,04 \\
\hline Hogares con dos fuentes de ingresos & 12,25 & 9,50 & 7,42 & 6,64 & 4,66 & 7,51 & 26,13 & 29,42 & 31,36 & 36,11 & 45,29 & 35,07 \\
\hline Hogares donde nadie trabaja CON niños & 15,27 & 11,00 & 10,06 & 8,00 & 9,54 & 13,94 & 24,72 & 17,98 & 16,38 & 14,32 & 19,28 & 22,72 \\
\hline Hogares donde nadie trabaja SIN niños & 17,98 & 11,49 & 10,14 & 8,62 & 7,86 & 10,19 & 32,10 & 19,00 & 16,92 & 14,54 & 14,83 & 17,35 \\
\hline Hogares donde nadie trabaja & 15,80 & 11,37 & 10,14 & 8,58 & 7,93 & 11,19 & 26,16 & 18,75 & 16,90 & 14,53 & 15,01 & 18,78 \\
\hline Total & 13,41 & 9,98 & 8,09 & 7,17 & 4,95 & 8,72 & 28,40 & 30,46 & 30,61 & 32,87 & 44,66 & 33,40 \\
\hline
\end{tabular}

Fuente: Elaboración propia, sobre la base de Instituto Nacional de Estadística y Censos (INDEC), Encuesta Nacional de Gastos de los Hogares 2012/2013. Resumen metodológico, Buenos Aires, 2013, y D. Rossignolo, "Gender equity in taxation in Argentina: the case of indirect and direct taxes", IDB Working Paper, Washington, D.C., Banco Interamericano de Desarrollo (BID), 2016, por aparecer. 